\title{
Observability of modally reduced order models with unknown parameters ${ }^{\star, \star \star}$
}

\author{
K. Maes ${ }^{\mathrm{a}, *}$, M.N. Chatzis ${ }^{\mathrm{b}}$, R. Vandebril ${ }^{\mathrm{c}}$, G. Lombaert $^{\mathrm{a}}$ \\ ${ }^{a} K U$ Leuven, Department of Civil Engineering, Leuven, Belgium \\ ${ }^{b}$ The University of Oxford, Department of Engineering Science, Oxford, United Kingdom \\ ${ }^{c} K U$ Leuven, Department of Computer Science, Leuven, Belgium
}

\begin{abstract}
Modally reduced order models are commonly adopted in system inversion. Their observability requires specific attention, since these models only accurately describe the dynamic behavior of the underlying system in a limited frequency range. This paper elaborates a methodology to investigate the observability of modally reduced order models with unknown parameters. The focus is on a particular type of model where the quasi-static contribution of the out-of-band modes is accounted for using so-called dummy modes. The observability test is performed by means of the commonly used Observability Rank Condition (ORC). The proposed methodology is illustrated by multiple examples from structural engineering. It is found that modally reduced order models serve as a valuable alternative for full order models when applied in system inversion. Not only are they computationally much less demanding, but due to their strong link with the underlying full order model, they also allow for the identification of physical parameters, such as mass or stiffness.
\end{abstract}

Keywords: system identification, geometric observability, identifiability, quasi-static correction

\section{Introduction}

The observability of time-invariant linear and nonlinear systems is of utmost importance when designing a sensor network for system inversion. The observability of a system, i.e., whether the unknown quantities (states, inputs, and parameters) can be estimated from a given set of measurements, is a theoretical property of the dynamic system examined under a specific sensor layout. If a state, an input, or a parameter is theoretically unobservable, it cannot be expected that any system identification method would be successful at properly estimating its value.

The observability of linear systems with known parameters is well understood [1]. For linear systems with unknown parameters, however, linear observability techniques cannot be straightforwardly applied, since the state augmentation performed in the case of unknown parameters results

\footnotetext{
${ }^{\star}$ Postprint submitted to Mechanical Systems and Signal Processing

${ }^{\star \star}$ Published version: K. Maes, M.N. Chatzis, R. Vandebril, and G. Lombaert. Observability of modally reduced order models with unknown parameters. Mechanical Systems and Signal Processing, 146:106993, 2021. https://doi.org/10.1016/j.ymssp.2020.106993

${ }^{*}$ Corresponding author. Tel.: +32 (0) 16322573.

Email address: kristof.maes@kuleuven.be (K. Maes)
} 
in a nonlinear system description [2]. Hence, it can be argued that all identification problems for systems with unknown parameters require the use of observability tools for nonlinear systems. In general, two different approaches are presented in the literature to deal with the theoretical observability of nonlinear systems, which are referred to as geometric approaches and algebraic approaches. Most geometric observability methods build on the work of Hermann and Krener [3], which extends the observability test for linear systems to nonlinear analytical systems by developing the Observability Rank Condition (ORC) based on Lie algebra $[4,5]$. On the other hand, algebraic observability methods have been developed for the special case of polynomial and rational systems $[6,7,8]$. The connection between geometric and algebraic observability is elaborated in [9].

The previously discussed observability methods assume the system input to be known and therefore do not cover the ability to track the system states in presence of unmeasured inputs and/or the ability to track these inputs [10]. Martinelli [11] has recently suggested an extension of the ORC to investigate the observability of the states of systems with partially measured inputs and for investigating the ability to simultaneously observe unmeasured inputs and their derivatives with respect to time. The resulting method is named the Extended ORC or EORC. A common assumption of the ORC and the EORC is that the measurements are not immediately affected by the inputs. To overcome this limitation, Maes et al. [12] have extended the ORC to study the observability of nonlinear analytical systems with partially measured inputs and outputs affected by a direct feedthrough. This extension is for example important for structural engineering applications where accelerations are measured in presence of unmeasured inputs. The resulting method is named the ORC-DF.

The application of observability methods to high-dimensional systems with unknown parameters often results in a significant computational cost, especially in terms of computer memory requirements [13]. Although specialized implementations of the ORC for linear systems with unmeasured parameters [14] and rational systems [8] allow for handling substantially larger systems, the application to real-life mechanical systems, often consisting of thousands of degrees of freedom, is still challenging, especially when the number of unkown parameters is large. In system inversion, full-order models are often replaced by computationally less expensive modally reduced order models (MROMs). For these MROMs, the dimensionality is generally much smaller and the application of standard observability methods becomes feasible. When applied to MROMs, however, a full symbolic evaluation of the observability test, as often required, is no longer possible, since a symbolic calculation of the modal properties of the system is infeasible. In addition, specific attention should be paid in the observability test due to the fact that MROMs only accurately describe the dynamic behavior of the underlying system in a limited frequency range.

This paper elaborates a methodology to investigate the observability of modally reduced order models with unknown parameters. The focus is on one particular type of model, proposed in [15], where the quasi-static contribution of the out-of-band modes is accounted for by means of socalled dummy modes. The observability test is performed here by means of the commonly used Observability Rank Condition (ORC) algorithm, but the developed framework is also applicable to other geometric and algebraic observability algorithms, including the EORC and the ORC-DF. It is explained how the frequency-dependent accuracy of the MROM affects the observability test. The resulting observability of the modally reduced order model, referred to as MROM-observability, enables investigating which combination of physical and modal parameters can be obtained from a system identification when applying a modally reduced order model.

The paper is outlined as follows. Section 2 presents the observability framework that applies to 
the case of modally reduced order models with quasi-static correction. Section 3 presents an illustration of the proposed methodology using suitably chosen examples from structural engineering. Finally, Section 4 concludes the work.

\section{Observability framework}

\subsection{State-space representation}

The dynamic behavior of a linear system $\mathcal{S}$ with time-invariant (unknown) parameters can in general be described by the following system of equations:

$$
\begin{aligned}
\dot{\mathbf{x}}_{\mathrm{t}} & =\mathbf{A}_{\mathrm{t}}(\boldsymbol{\theta}) \mathbf{x}_{\mathrm{t}}+\mathbf{B}_{\mathrm{t}}(\boldsymbol{\theta}) \mathbf{u} \\
\mathbf{y} & =\mathbf{G}_{\mathrm{t}}(\boldsymbol{\theta}) \mathbf{x}_{\mathrm{t}}+\mathbf{J}(\boldsymbol{\theta}) \mathbf{u}
\end{aligned}
$$

where $\mathbf{x}_{\mathrm{t}}$ is the state vector of size $n_{\mathrm{St}}, \mathbf{u}$ is the input vector of size $n_{\mathrm{u}}, \boldsymbol{\theta}$ is the vector of parameters of size $n_{\theta}$, and $\mathbf{y}$ is the output vector of size $n_{\mathrm{y}}$. In what follows, it is assumed that all inputs $\mathbf{u}$ are measured and hence known. This corresponds to the case where the number of unmeasured inputs, referred to as $n_{\mathrm{p}}$, equals zero. The developed framework also applies to the more general case where some or all of the inputs are unknown $\left(n_{\mathrm{p}}>0\right)$. This, however, requires the application of alternative observability techniques, such as the EORC [11] or the ORC-DF [12], which are beyond the scope of this paper.

Augmenting the state vector $\mathbf{x}_{\mathrm{t}}$ with the vector of time-invariant parameters $\boldsymbol{\theta}$, with $\dot{\boldsymbol{\theta}}=\mathbf{0}$, allows rewriting system (1-2) in its augmented form:

$$
\begin{aligned}
& \dot{\mathbf{x}}=\mathbf{A}(\boldsymbol{\theta}) \mathbf{x}+\mathbf{B}(\boldsymbol{\theta}) \mathbf{u} \\
& \mathbf{y}=\mathbf{G}(\boldsymbol{\theta}) \mathbf{x}+\mathbf{J}(\boldsymbol{\theta}) \mathbf{u}
\end{aligned}
$$

where $\mathbf{x}=\left[\begin{array}{ll}\mathbf{x}_{\mathrm{t}}^{\mathrm{T}} & \boldsymbol{\theta}^{\mathrm{T}}\end{array}\right]^{\mathrm{T}}$ and the matrices $\mathbf{A}, \mathbf{B}$, and $\mathbf{G}$ are given by:

$$
\mathbf{A}=\left[\begin{array}{cc}
\mathbf{A}_{\mathrm{t}} & \mathbf{0} \\
\mathbf{0} & \mathbf{0}
\end{array}\right], \quad \mathbf{B}=\left[\begin{array}{c}
\mathbf{B}_{\mathrm{t}} \\
\mathbf{0}
\end{array}\right], \quad \mathbf{G}=\left[\begin{array}{ll}
\mathbf{G}_{\mathrm{t}} & \mathbf{0}
\end{array}\right]
$$

The matrix $\mathbf{J}$ is not affected by the state augmentation. The model described by Eqs. (3-4) is hereafter referred to as $\mathcal{M}$. Note that (3-4) is an affine-input state-space representation.

The aim of observability methods is to investigate the observability of the dynamic system states $\mathbf{x}_{\mathrm{t}}$ and the identifiability of the time-invariant parameters $\boldsymbol{\theta}$. The observability test is performed under the assumption that the model $\mathcal{M}$ accurately represents the dynamic behavior of the system $\mathcal{S}$.

\subsection{Modally reduced order models with quasi-static correction}

A numerical model representing a civil or mechanical engineering structure is usually constructed to reproduce its dynamic behavior in a particular frequency range with finite bandwidth. This will determine i.a. the level of detail and the element size adopted in the space-discretization of the continuous system, for example, when adopting the finite element method. Within the frequency range of interest, the number of modes that significantly contributes to the dynamic response is generally much lower than the total number of degrees of freedom that characterizes the 
(full order) numerical model. For most applications, the use of a reduced modal basis, a.k.a. model order reduction, leads to a significant reduction of the computational effort when performing numerical simulations, meanwhile not jeopardizing the prediction accuracy of the model.

It was shown by Maes and Lombaert [15] that it is essential to account for the quasi-static contribution of the so-called out-of-band modes when adopting a modally reduced order model for system inversion. When not accounted for, large errors in the estimated inputs, states and/or parameters may occur. In the same paper [15], the authors have developed a technique that enables quasi-static correction when applying model order reduction in combination with statespace modeling. This technique accounts for the quasi-static contribution of the out-of-band modes by means of additional so-called dummy modes. The number of additional modes equals the number of forces applied to the structure $n_{\mathrm{u}}$ and is therefore generally low. As such, the increase in computational effort due to the quasi-static correction is low as well. The following example is taken from [15] and is provided here to illustrate the proposed model correction technique.

Example 2.1. Consider the case of a simply supported steel beam (Fig. 1) with a length of $1 \mathrm{~m}$ and a rectangular cross section of width $50.8 \mathrm{~mm}$ and height $25.4 \mathrm{~mm}$. The Young's modulus, Poisson's ratio, and material density are taken as $210 \mathrm{GPa}, 0.3$, and $7750 \mathrm{~kg} / \mathrm{m}^{3}$, respectively. Ideal hinges are assumed at both ends of the beam, only constraining the translation of the end nodes and not their rotation.

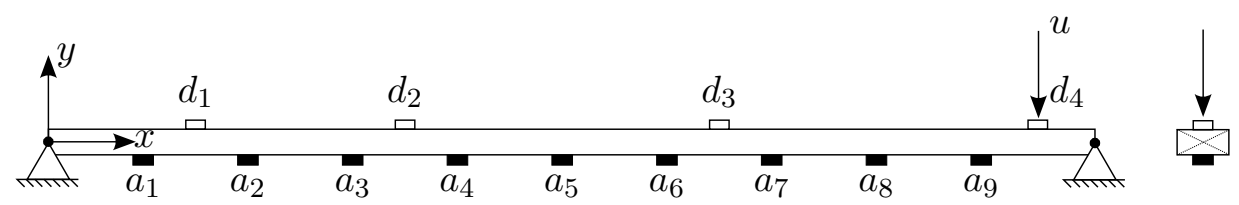

Fig. 1: Side view (left) and cross section (right) of a simply supported steel beam, indicating the force $u$ and possible response measurements locations ( $a_{i}$ : vertical acceleration $i, d_{i}$ : vertical displacement $i$ ).

A two-dimensional finite element (FE) model of the beam with 100 Euler-Bernoulli beam elements is constructed. Only bending in the vertical plane is considered. Shear deformation is not accounted for. The first four natural frequencies obtained from the beam model are $59.9 \mathrm{~Hz}$, $239.6 \mathrm{~Hz}, 538.3 \mathrm{~Hz}$, and $955.2 \mathrm{~Hz}$. A modal damping ratio of $2.5 \%$ is assumed for all modes.

The excitation of the beam consists of a single vertical force $u$, applied at a distance of $0.05 \mathrm{~m}$ from the right support. The force location is chosen close to the support to have a significant contribution of the higher modes. In the considered case, the focus is put on the frequency range from 0 to $1000 \mathrm{~Hz}$.

Fig. 2 compares the transfer functions $H_{\mathrm{yu}}(\omega), H_{\mathrm{yu}}^{\prime}(\omega)$, and $H_{\mathrm{yu}}^{\prime \prime}(\omega)$ which relate displacement $d_{4}$ to the force $u$. Transfer function $H_{\mathrm{yu}}(\omega)$ represents the full order model $\mathcal{M}_{0}$ that is obtained by including all modes in the model (200 modes). Transfer function $H_{\mathrm{yu}}^{\prime}(\omega)$ represents a model $\mathcal{M}_{1}$ that is obtained after model order reduction, hereby only retaining the first four vertical bending modes of the beam, i.e. all modes with a natural frequency in the frequency range of interest $(0$ to $1000 \mathrm{~Hz}$ ). The contribution of the other modes is not accounted for. Finally, transfer function $H_{\text {yu }}^{\prime \prime}(\omega)$ represents a model $\mathcal{M}_{2}$ that is obtained after model order reduction, where in addition to the dynamic contribution of the first four vertical bending modes the quasi-static contribution of the higher modes is accounted for by means of a single dummy mode. The natural frequency and modal damping ratio of the dummy mode are assigned a value of $1800 \mathrm{~Hz}$ and $2 \%$, respectively. This choice of the dummy mode parameters results in a nearly constant (i.e. quasi-static) contribution 
of the dummy mode to the transfer function in the frequency range between 0 and $1000 \mathrm{~Hz}$. The reader is referred to [15] for an in depth discussion of the choice of the dummy mode parameters.

(a)

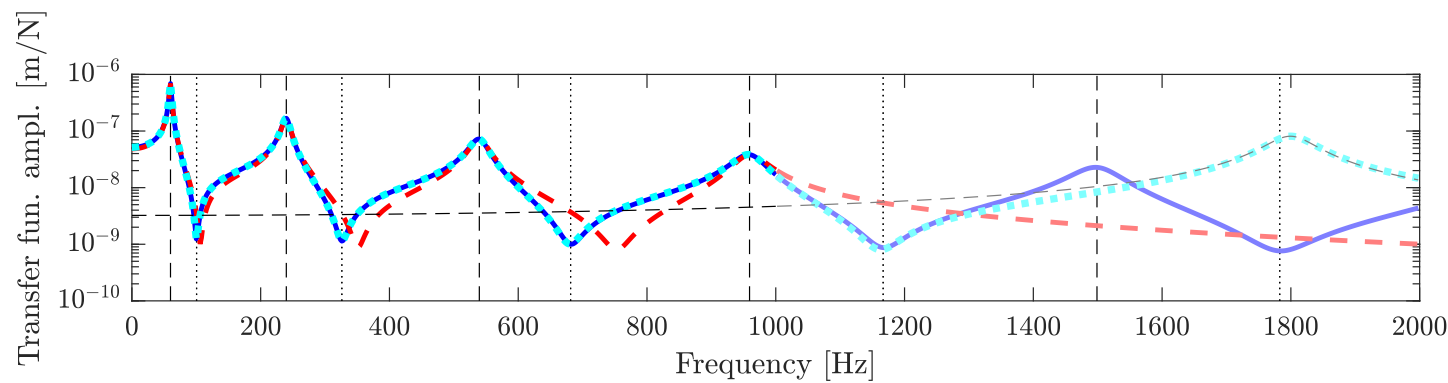

(b)

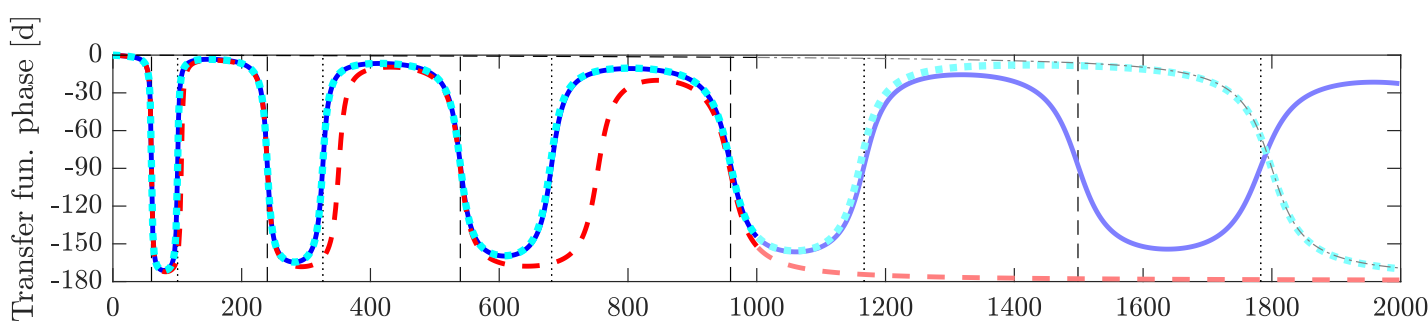

Fig. 2: (a) Amplitude and (b) phase of transfer functions $H_{\mathrm{yu}}(\omega)$ (200 modes, blue solid line), $H_{\mathrm{yu}}^{\prime}(\omega)$ (4 modes, red dashed line), and $H_{\mathrm{yu}}^{\prime \prime}(\omega)$ (4 modes with dummy mode correction, cyan dotted line) that relate displacement $d_{4}$ to force $u$. The contribution of the dummy mode is indicated by a black dashed line. The undamped natural frequencies are indicated by vertical dashed lines, the antiresonance frequencies of the full order model by vertical dotted lines.

Transfer functions $H_{\mathrm{yu}}(\omega)$ and $H_{\mathrm{yu}}^{\prime}(\omega)$ nearly coincide at resonance, where a single mode dominates the response of the system. A large difference between the transfer functions is observed near antiresonance and in the quasi-static frequency range, where higher modes that are omitted in model $\mathcal{M}_{1}$ significantly contribute to the system response. Comparison of transfer functions $H_{\mathrm{yu}}(\omega)$ and $H_{\mathrm{yu}}^{\prime \prime}(\omega)$ shows a very good overall accuracy of model $\mathcal{M}_{2}$ in the frequency range from 0 to $1000 \mathrm{~Hz}$, while only requiring 5 modes (4 structural modes and 1 dummy mode), i.e., only 1 more than model $\mathcal{M}_{1}$.

As illustrated in example 2.1, a modally reduced order model with quasi-static correction can serve as a computationally efficient and accurate representation of a high-dimensional linear system. When used in system inversion, the key question lies in the observability of such a model and, particularly, how the observability is affected by the limited frequency range for which the model accurately describes the true system behavior. This is the topic of the following sections.

\subsection{System matrices}

The expressions for the system matrices in the case where dummy modes are included for quasi-static correction have been derived in [15] and are here repeated for the sake of convenience.

Consider the case where the full dynamic contribution of $n_{\mathrm{m}}$ modes is accounted for in the model. In general, these consist of the modes with a natural frequency within the frequency range of interest, which is determined by the application. In addition, the quasi-static contribution of 
the remaining (out-of-band) modes is accounted for by $n_{\mathrm{u}}$ dummy modes, where $n_{\mathrm{u}}$ equals the number of independent inputs to the system. The dynamic state vector of the dummy mode model $\mathbf{x}_{\mathrm{t}} \in \mathbb{R}^{2\left(n_{\mathrm{m}}+n_{\mathrm{u}}\right)}$ contains the modal displacements $\mathbf{z} \in \mathbb{R}^{n_{\mathrm{m}}}$ and modal velocities $\dot{\mathbf{z}} \in \mathbb{R}^{n_{\mathrm{m}}}$ corresponding to the $n_{\mathrm{m}}$ structural modes, as well as the modal displacements $\mathbf{z}^{\prime} \in \mathbb{R}^{n_{\mathrm{u}}}$ and modal velocities $\dot{\mathbf{z}}^{\prime} \in \mathbb{R}^{n_{\mathrm{u}}}$ corresponding to the $n_{\mathrm{u}}$ dummy modes, respectively:

$$
\mathbf{x}_{\mathrm{t}}=\left[\begin{array}{c}
\mathbf{z} \\
\mathbf{z}^{\prime} \\
\dot{\mathbf{z}} \\
\dot{\mathbf{z}}^{\prime}
\end{array}\right]
$$

The system matrices $\mathbf{A}_{\mathrm{t}}$ and $\mathbf{B}_{\mathrm{t}}$ are given by:

$$
\mathbf{A}_{\mathrm{t}}=\left[\begin{array}{cccc}
\mathbf{0} & \mathbf{0} & \mathbf{I}_{n_{\mathrm{m}}} & \mathbf{0} \\
\mathbf{0} & \mathbf{0} & \mathbf{0} & \mathbf{I}_{n_{\mathrm{u}}} \\
-\mathbf{\Omega}^{2} & \mathbf{0} & -\mathbf{C}_{\mathrm{m}} & \mathbf{0} \\
\mathbf{0} & -\mathbf{\Omega}^{\prime 2} & \mathbf{0} & -\boldsymbol{\Gamma}^{\prime}
\end{array}\right], \quad \mathbf{B}_{\mathrm{t}}=\left[\begin{array}{c}
\mathbf{0} \\
\mathbf{0} \\
\boldsymbol{\Phi}^{\mathrm{T}} \mathbf{S}_{\mathrm{u}} \\
\boldsymbol{\Phi}_{\mathrm{u}}^{\prime}
\end{array}\right]
$$

where $\boldsymbol{\Omega} \in \mathbb{R}^{n_{\mathrm{m}} \times n_{\mathrm{m}}}$ is a diagonal matrix containing the natural frequencies $\omega_{m}\left(m=1, \ldots, n_{\mathrm{m}}\right)$ on its diagonal $\left((\boldsymbol{\Omega})_{m m}=\omega_{m}\right)$, with $n_{\mathrm{m}}$ the number of modes accounted for in the system model. The matrix $\boldsymbol{\Omega}^{\prime} \in \mathbb{R}^{n_{\mathrm{u}} \times n_{\mathrm{u}}}$ is a diagonal matrix containing the terms $\omega_{n}^{\prime}$ on its diagonal $\left(\left(\boldsymbol{\Omega}^{\prime}\right)_{n n}=\omega_{n}^{\prime}\right)$, with $\omega_{n}^{\prime}$ the natural frequency assigned to dummy mode $n$. The matrix $\mathbf{C}_{\mathrm{m}} \in \mathbb{R}^{n_{\mathrm{m}} \times n_{\mathrm{m}}}$ is the modal damping matrix, given by $\mathbf{C}_{\mathrm{m}}=\boldsymbol{\Phi}^{\mathrm{T}} \mathbf{C}_{\mathrm{v}} \boldsymbol{\Phi}$, where $\mathbf{C}_{\mathrm{v}} \in \mathbb{R}^{n_{\mathrm{dof}} \times n_{\mathrm{dof}}}$ is the viscous damping matrix and $\boldsymbol{\Phi} \in \mathbb{R}^{n_{\mathrm{dof}} \times n_{\mathrm{m}}}$ is the matrix which contains the mass normalized mode shapes $\phi_{m} \in \mathbb{R}^{n_{\mathrm{dof}}}$ as columns $\left((\boldsymbol{\Phi})_{: m}=\phi_{m}\right)$, with $n_{\text {dof }}$ the number of degrees of freedom. In the case of proportional damping, $\mathbf{C}_{\mathrm{m}}=\boldsymbol{\Gamma}$, where $\boldsymbol{\Gamma}$ is a diagonal matrix containing the terms $2 \xi_{m} \omega_{m}$ on its diagonal $\left((\boldsymbol{\Gamma})_{m m}=2 \xi_{m} \omega_{m}\right)$, with $\xi_{m}$ the modal damping ratio corresponding to mode $m$. The matrix $\boldsymbol{\Gamma}^{\prime} \in \mathbb{R}^{n_{\mathrm{u}} \times n_{\mathrm{u}}}$ is a diagonal matrix containing the terms $2 \xi_{n}^{\prime} \omega_{n}^{\prime}$ on its diagonal $\left(\left(\boldsymbol{\Gamma}^{\prime}\right)_{n n}=2 \xi_{n}^{\prime} \omega_{n}^{\prime}\right)$, with $\xi_{n}^{\prime}$ the modal damping ratio assigned to dummy mode $n$. The matrices $\mathbf{I}_{n_{\mathrm{m}}} \in \mathbb{R}^{n_{\mathrm{m}} \times n_{\mathrm{m}}}$ and $\mathbf{I}_{n_{\mathrm{u}}} \in \mathbb{R}^{n_{\mathrm{u}} \times n_{\mathrm{u}}}$ denote identity matrices and $\mathbf{S}_{\mathrm{u}} \in \mathbb{R}^{n_{\mathrm{dof}} \times n_{\mathrm{u}}}$ is a selection matrix specifying the input locations. Finally, $\boldsymbol{\Phi}_{\mathrm{u}}^{\prime} \in \mathbb{R}^{n_{\mathrm{u}} \times n_{\mathrm{u}}}$ is a diagonal matrix containing on its diagonal the dummy mode shape components at the input locations, $\phi_{\mathrm{u} n}^{\prime}$, which are computed as:

$$
\phi_{\mathrm{u} n}^{\prime}=\omega_{\mathrm{u} n}^{\prime} \sqrt{\mathbf{S}_{\mathrm{u}_{n}}^{\mathrm{T}} \mathbf{K}^{-1} \mathbf{S}_{\mathrm{u}_{n}}-\sum_{m=1}^{n_{\mathrm{m}}} \frac{\mathbf{S}_{\mathrm{u}_{n}}^{\mathrm{T}} \boldsymbol{\phi}_{m} \boldsymbol{\phi}_{m}^{\mathrm{T}} \mathbf{S}_{\mathrm{u}_{n}}}{\omega_{m}^{2}}}
$$

where $\mathbf{S}_{\mathrm{u}_{n}}$ represents the $n$-th column of the selection matrix $\mathbf{S}_{u}$ and $\mathbf{K} \in \mathbb{R}^{n_{\text {dof }} \times n_{\text {dof }}}$ is the stiffness matrix of the structural model. The natural frequency and modal damping ratio assigned to the dummy modes are generally chosen such that the contribution of all dummy modes to the frequency range of interest is nearly constant. These parameters are independent of the actual underlying system. The reader is referred to [15] for an in-depth discussion on the choice of the natural frequency and modal damping ratio assigned to the dummy modes. Finally, note that the dummy modes are assumed to be mutually decoupled and also decoupled from the $n_{\mathrm{m}}$ structural modes.

The expressions for the system matrices $\mathbf{G}_{\mathrm{t}}$ and $\mathbf{J}$ are given by:

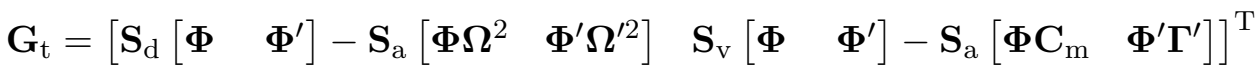

$$
\begin{aligned}
& \mathbf{J}=\mathbf{S}_{\mathrm{a}} \boldsymbol{\Phi} \boldsymbol{\Phi}^{\mathrm{T}} \mathbf{S}_{\mathrm{u}}+\mathbf{S}_{\mathrm{a}} \boldsymbol{\Phi}^{\prime} \boldsymbol{\Phi}_{\mathrm{u}}^{\prime}
\end{aligned}
$$


The matrices $\mathbf{S}_{\mathrm{a}} \in \mathbb{R}^{n_{\mathrm{y}} \times n_{\text {dof }}}, \mathbf{S}_{\mathrm{v}} \in \mathbb{R}^{n_{\mathrm{y}} \times n_{\text {dof }}}$, and $\mathbf{S}_{\mathrm{d}} \in \mathbb{R}^{n_{\mathrm{y}} \times n_{\text {dof }}}$ are selection matrices indicating the degrees of freedom corresponding to the acceleration, velocity, and displacement or strain measurements, respectively. The matrix $\boldsymbol{\Phi}^{\prime} \in \mathbb{R}^{n_{\mathrm{dof}} \times n_{\mathrm{u}}}$ is a matrix containing as columns the dummy mode shape vectors $\phi_{\mathrm{d} n}^{\prime} \in \mathbb{R}^{n_{\mathrm{dof}}}$, which are computed as:

$$
\phi_{\mathrm{d} n}^{\prime}=\frac{\omega_{n}^{\prime 2}}{\phi_{\mathrm{u} n}^{\prime}}\left(\mathbf{K}^{-1} \mathbf{S}_{\mathrm{u}_{n}}-\sum_{m=1}^{n_{\mathrm{m}}} \frac{\phi_{m} \boldsymbol{\phi}_{m}^{\mathrm{T}} \mathbf{S}_{\mathrm{u}_{n}}}{\omega_{m}^{2}}\right)
$$

Note that $\phi_{\mathrm{u} n}^{\prime}=\mathbf{S}_{\mathrm{u}_{n}}^{\mathrm{T}} \phi_{\mathrm{d} n}^{\prime}$.

\subsection{The Observability Rank Condition}

The commonly adopted ORC algorithm is well-suited to investigate the observability of the modally reduced order model with quasi-static correction presented in Sections 2.2 and 2.3. This section briefly recapitulates the ORC algorithm as presented in $[4,5]$. In addition, it is shown how the implementation of the algorithm can be adjusted for the considered case of a linear underlying system with unknown parameters. The adjustment allows for a significant speed-up of the observability test.

In general, the ORC algorithm presented in [4] considers the following analytic and affine-input system description:

$$
\dot{\mathbf{x}}=\boldsymbol{f}(\mathbf{x})+\sum_{j=1}^{n_{\mathrm{u}}} \boldsymbol{g}_{j}(\mathbf{x}) u_{j}, \quad \mathbf{y}=\boldsymbol{h}_{0}(\mathbf{x})
$$

The previously considered system description in Eqs. (3-4) is a special case of the system description in Eq. (12), with:

$$
\boldsymbol{f}(\mathbf{x})=\mathbf{A}(\boldsymbol{\theta}) \mathbf{x}, \quad \boldsymbol{g}_{j}(\mathbf{x})=\mathbf{B}_{j}(\boldsymbol{\theta}), \quad \text { and } \boldsymbol{h}_{0}(\mathbf{x})=\mathbf{G}(\boldsymbol{\theta}) \mathbf{x}
$$

where $\mathbf{B}_{j}$ denotes the $j$-th column of the matrix $\mathbf{B}$ corresponding to input $u_{j}$. The system description in Eq. (12) considers all inputs $u_{j}$ to be known $\left(n_{\mathrm{p}}=0\right)$ and does not consider a direct feedthrough from these inputs $u_{j}$ to the system response $\mathbf{y}$ (i.e. $\mathbf{J}=\mathbf{0}$ ). Note that the latter assumption restricts the use of the ORC to displacement, strain, and velocity measurements, which do not result in a direct feedthrough in the output equation. When accelerations are measured, such a direct feedthrough is inherently present. In this case, one should use the recently developed ORC-DF [12] instead of the original ORC algorithm. The presented methodology, however, remains valid and can be readily extended.

A basic tool used in the ORC algorithm is the Lie derivative. The Lie derivative of a vector of $n_{\mathrm{o}}$ scalar functions $\mathcal{O}=\left[\begin{array}{lll}o_{1}(\mathbf{x}) & \ldots & o_{n_{\mathrm{o}}}(\mathbf{x})\end{array}\right]^{\mathrm{T}}$ along a vector field $\boldsymbol{\nu}=\left[\begin{array}{lll}\nu_{1} & \ldots & \nu_{n_{\mathrm{s}}}\end{array}\right]^{\mathrm{T}}$ is defined as:

$$
L_{\boldsymbol{\nu}}(\mathcal{O})=\mathrm{d} \mathcal{O} \cdot \boldsymbol{\nu}
$$

where $\mathrm{d} \mathcal{O}$ is defined as:

$$
\mathrm{d} \mathcal{O}=\left[\begin{array}{c}
\nabla o_{1}(\mathbf{x}) \\
\vdots \\
\nabla o_{n_{\mathrm{o}}}(\mathbf{x})
\end{array}\right]
$$


and $\nabla$ denotes the gradient of a scalar function with respect to the vector $\mathbf{x}$ and $\cdot$ in Eq. (14) stands for the post-multiplication of the matrix $\mathrm{d} \mathcal{O}$ with the vector $\boldsymbol{\nu}$.

The steps of the ORC algorithm are outlined as follows [2]:

1. Starting point: $k=0, \mathcal{O}_{0}=\boldsymbol{h}_{0}, \Delta \mathcal{O}_{0}=\mathcal{O}_{0}$.

2. Calculate $\mathrm{d} \mathcal{O}_{0}=\frac{\partial \mathcal{O}_{0}}{\partial \mathbf{x}}$.

3. Calculate $\Delta \boldsymbol{\mathcal { O }}_{k+1}=\left[\begin{array}{llll}\left(L_{\boldsymbol{f}}\left(\Delta \mathcal{O}_{k}\right)\right)^{\mathrm{T}} & \left(L_{\boldsymbol{g}_{1}}\left(\Delta \mathcal{O}_{k}\right)\right)^{\mathrm{T}} & \ldots & \left(L_{\boldsymbol{g}_{n_{\mathrm{u}}}}\left(\Delta \boldsymbol{\mathcal { O }}_{k}\right)\right)^{\mathrm{T}}\end{array}\right]^{\mathrm{T}}$.

4. Calculate $\mathrm{d} \Delta \mathcal{O}_{k+1}=\frac{\partial \Delta \mathcal{O}_{k+1}}{\partial \mathbf{x}}$.

5. Calculate $\mathrm{d} \mathcal{O}_{k+1}=\left[\begin{array}{ll}\mathrm{d} \mathcal{O}_{k}^{\mathrm{T}} & \mathrm{d} \Delta \mathcal{O}_{k+1}^{\mathrm{T}}\end{array}\right]^{\mathrm{T}}$.

6. If $\operatorname{rank}\left(\mathrm{d} \mathcal{O}_{k+1}\right)=\operatorname{rank}\left(\mathrm{d} \mathcal{O}_{k}\right)$, or $\operatorname{rank}\left(\mathrm{d} \mathcal{O}_{k+1}\right)=n_{\mathrm{s}}$, or $k=n_{\mathrm{s}}-2$, end.

7. $k=k+1$ and go to step 3 .

After the algorithm has come to an end, the ORC is satisfied if and only if $\operatorname{rank}\left(\mathrm{d} \mathcal{O}_{k+1}\right)=n_{\mathrm{s}}$, with $n_{\mathrm{s}}$ the dimension of the (augmented) state vector $\mathbf{x}$. If the ORC is satisfied, all states $\mathbf{x}$ are theoretically observable, meaning that the dynamic states $\mathbf{x}_{\mathrm{t}}$ may be tracked over time and that the time-invariant parameters $\boldsymbol{\theta}$ may be identified from the system response $\mathbf{y}$ [2]. If $\operatorname{rank}\left(\mathrm{d} \mathcal{O}_{k+1}\right)<n_{\mathrm{s}}$, the system is not observable. However, in this case, it is desirable from an identification point of view to investigate the partial observability for each of the states separately. A method to investigate the partial observability of a system is discussed by Chatzis et al. in [2]. In this method, the $l^{\text {th }}$ column of the matrix $\mathrm{d} \mathcal{O}_{k+1}$ is removed and the rank of the occurring matrix $\mathrm{d} \mathcal{O}_{k+1}^{l}$ is computed. If $\operatorname{rank}\left(\mathrm{d} \mathcal{O}_{k+1}^{l}\right)<\operatorname{rank}\left(\mathrm{d} \mathcal{O}_{k+1}\right)$, the $l^{\text {th }}$ state is observable. Otherwise, it is not. This allows distinguishing between the observable and unobservable states in every step $k$. Finally, note that if the $l^{\text {th }}$ state is observable for a given step $k$, it will also be observable for all successive steps $k+q$, with $q>0$ and one can exclude the $l^{\text {th }}$ state in the partial observability test to avoid redundant calculations.

For the considered case of a linear underlying system with unknown parameters, the calculation of $\Delta \mathcal{O}_{k+1}$ in step 3 and $\mathrm{d} \Delta \mathcal{O}_{k+1}$ in step 4 can be simplified, for example as shown by Shi et al. in [14]. Indeed, for the model given in Eqs. (12) and (13), it is derived that:

$$
L_{\boldsymbol{f}}\left(\Delta \mathcal{O}_{k}\right)=\left[\begin{array}{c}
L_{\boldsymbol{f}}\left(\mathbf{S}_{\mathrm{t}} \Delta \mathcal{O}_{k}\right) \\
\mathbf{0}
\end{array}\right], \quad L_{\boldsymbol{g}_{j}}\left(\Delta \mathcal{O}_{k}\right)=\left[\begin{array}{c}
L_{\boldsymbol{g}_{j}}\left(\mathbf{S}_{\mathrm{t}} \Delta \mathcal{O}_{k}\right) \\
\mathbf{0}
\end{array}\right], \quad j=1, \ldots, n_{\mathrm{u}}
$$

where $\mathbf{S}_{\mathrm{t}}=\left[\begin{array}{ll}\mathbf{I}_{n_{\mathrm{y}}} & \mathbf{0}\end{array}\right]$, is a matrix that selects the first $n_{\mathrm{y}}$ rows of the matrix $\Delta \mathcal{O}_{k}$, with $\mathbf{I}_{n_{\mathrm{y}}} \in \mathbb{R}^{n_{\mathrm{y}}}$ the identity matrix. Since the zero rows in the matrix $\Delta \mathcal{O}_{k+1}$ do not add information, they can be omitted. The calculation of $\Delta \mathcal{O}_{k+1}$ in step 3 can therefore be replaced by:

$$
\Delta \mathcal{O}_{k+1}=\left[\begin{array}{llll}
\left(L_{\boldsymbol{f}}\left(\mathbf{S}_{\mathrm{t}} \Delta \mathcal{O}_{k}\right)\right)^{\mathrm{T}} & \left(L_{\boldsymbol{g}_{1}}\left(\mathbf{S}_{\mathrm{t}} \Delta \mathcal{O}_{k}\right)\right)^{\mathrm{T}} \quad \ldots \quad\left(L_{\boldsymbol{g}_{n_{\mathrm{u}}}}\left(\mathbf{S}_{\mathrm{t}} \Delta \mathcal{O}_{k}\right)\right)^{\mathrm{T}}
\end{array}\right]^{\mathrm{T}}
$$

In addition, the Lie derivatives $L_{\boldsymbol{f}}\left(\mathbf{S}_{\mathrm{t}} \Delta \mathcal{O}_{k}\right)$ and $L_{\boldsymbol{g}_{j}}\left(\mathbf{S}_{\mathrm{t}} \Delta \mathcal{O}_{k}\right), j=1, \ldots, n_{\mathrm{u}}$ can be further elaborated as:

$$
\begin{aligned}
L_{\boldsymbol{f}}\left(\mathbf{S}_{\mathrm{t}} \Delta \mathcal{O}_{k}\right) & =\mathbf{G}_{\mathrm{t}} \mathbf{A}_{\mathrm{t}}^{k+1} \mathbf{x}_{\mathrm{t}} \\
L_{\boldsymbol{g}_{j}}\left(\mathbf{S}_{\mathrm{t}} \Delta \mathcal{O}_{k}\right) & =\mathbf{G}_{\mathrm{t}} \mathbf{A}_{\mathrm{t}}^{k} \mathbf{B}_{\mathrm{t}_{j}}
\end{aligned}
$$


Following Eq. (17), the Jacobian $\mathrm{d} \Delta \mathcal{O}_{k+1}$ in step 4 can be alternatively calculated as:

$$
\begin{aligned}
& \mathrm{d} \Delta \boldsymbol{\mathcal { O }}_{k+1}=\left[\begin{array}{ll}
\frac{\partial L_{\boldsymbol{f}}\left(\mathbf{S}_{\mathrm{t}} \Delta \boldsymbol{\mathcal { O }}_{k}\right)}{\partial \mathbf{x}_{\mathrm{t}}} & \frac{\partial L_{\boldsymbol{f}}\left(\mathbf{S}_{\mathrm{t}} \Delta \mathcal{O}_{k}\right)}{\partial \boldsymbol{\theta}}
\end{array}\right]^{\mathrm{T}} \quad \ldots \\
& \left.\left[\begin{array}{lll}
\frac{\partial L_{\boldsymbol{g}_{1}}\left(\mathbf{S}_{\mathrm{t}} \Delta \mathcal{O}_{k}\right)}{\partial \mathbf{x}_{\mathrm{t}}} & \frac{\partial L_{\boldsymbol{g}_{1}}\left(\mathbf{S}_{\mathrm{t}} \Delta \mathcal{O}_{k}\right)}{\partial \boldsymbol{\theta}}
\end{array}\right]^{\mathrm{T}} \quad \cdots \quad\left[\begin{array}{ll}
\frac{\partial L_{\boldsymbol{g}_{n_{\mathfrak{u}}}}\left(\mathbf{S}_{\mathrm{t}} \Delta \mathcal{O}_{k}\right)}{\partial \mathbf{x}_{\mathrm{t}}} & \frac{\partial L_{\boldsymbol{g}_{n_{\mathfrak{u}}}}\left(\mathbf{S}_{\mathrm{t}} \Delta \mathcal{O}_{k}\right)}{\partial \boldsymbol{\theta}}
\end{array}\right]^{\mathrm{T}}\right]^{\mathrm{T}}
\end{aligned}
$$

with

$$
\begin{aligned}
& \frac{\partial L_{\boldsymbol{f}}\left(\mathbf{S}_{\mathrm{t}} \Delta \mathcal{O}_{k}\right)}{\partial \mathbf{x}_{\mathrm{t}}}=\mathbf{G}_{\mathrm{t}} \mathbf{A}_{\mathrm{t}}^{k+1} \\
& \frac{\partial L_{\boldsymbol{f}}\left(\mathbf{S}_{\mathrm{t}} \Delta \boldsymbol{O}_{k}\right)}{\partial \theta_{q}}=\left(\frac{\partial \mathbf{G}_{\mathrm{t}}}{\partial \theta_{q}} \mathbf{A}_{\mathrm{t}}^{k+1}+\mathbf{G}_{\mathrm{t}} \sum_{q=0}^{k} \mathbf{A}_{\mathrm{t}}^{q} \frac{\partial \mathbf{A}_{\mathrm{t}}}{\partial \theta_{q}} \mathbf{A}_{\mathrm{t}}^{k-q}\right) \mathbf{x}_{\mathrm{t}} \\
& \frac{\partial L_{\boldsymbol{g}_{j}}\left(\mathbf{S}_{\mathrm{t}} \Delta \boldsymbol{O}_{k}\right)}{\partial \mathbf{x}_{\mathrm{t}}}=\mathbf{0} \\
& \frac{\partial L_{\boldsymbol{g}_{j}}\left(\mathbf{S}_{\mathrm{t}} \Delta \boldsymbol{O}_{k}\right)}{\partial \theta_{q}}=\frac{\partial \mathbf{G}_{\mathrm{t}}}{\partial \theta_{q}} \mathbf{A}_{\mathrm{t}}^{k} \mathbf{B}_{\mathrm{t}_{j}}+\mathbf{G}_{\mathrm{t}} \sum_{q=0}^{k-1} \mathbf{A}_{\mathrm{t}}^{q} \frac{\partial \mathbf{A}_{\mathrm{t}}}{\partial \theta_{q}} \mathbf{A}_{\mathrm{t}}^{k-q-1} \mathbf{B}_{\mathrm{t}_{j}}+\mathbf{G}_{\mathrm{t}} \mathbf{A}_{\mathrm{t}}^{k} \frac{\partial \mathbf{B}_{\mathrm{t}_{j}}}{\partial \theta_{q}}
\end{aligned}
$$

where $q=1, \ldots, n_{\theta}$. For the particular case of modal models with quasi-static correction, the computation of the derivatives of the system matrices $\mathbf{A}_{\mathrm{t}}, \mathbf{B}_{\mathrm{t}}$, and $\mathbf{G}_{\mathrm{t}}$ with respect to the parameters $\theta_{q}$ is addressed in Section 2.5. Taking into account the aforementioned considerations, the ORC method for the case of a linear system model with unknown parameters can be alternatively formulated as follows:

1. Starting point: $k=0, \mathbf{V}_{0}=\mathbf{G}_{\mathrm{t}}, \mathbf{W}_{0}^{q}=\frac{\partial \mathbf{G}_{\mathrm{t}}}{\partial \theta_{q}}, q=1, \ldots, n_{\theta}$.

2. Compose $\mathrm{d} \mathcal{O}_{0}=\left[\begin{array}{lllll}\mathbf{V}_{0} & \mathbf{W}_{0}^{1} \mathbf{x}_{\mathrm{t}} & \ldots & \mathbf{W}_{0}^{n_{\theta}} \mathbf{x}_{\mathrm{t}}\end{array}\right]$.

3. Calculate $\mathbf{V}_{k+1}=\mathbf{V}_{k} \mathbf{A}_{\mathrm{t}}, \mathbf{W}_{k+1}^{q}=\mathbf{W}_{k}^{q} \mathbf{A}_{\mathrm{t}}+\mathbf{V}_{k} \frac{\partial \mathbf{A}_{\mathrm{t}}}{\partial \theta_{q}}, q=1, \ldots, n_{\theta}$.

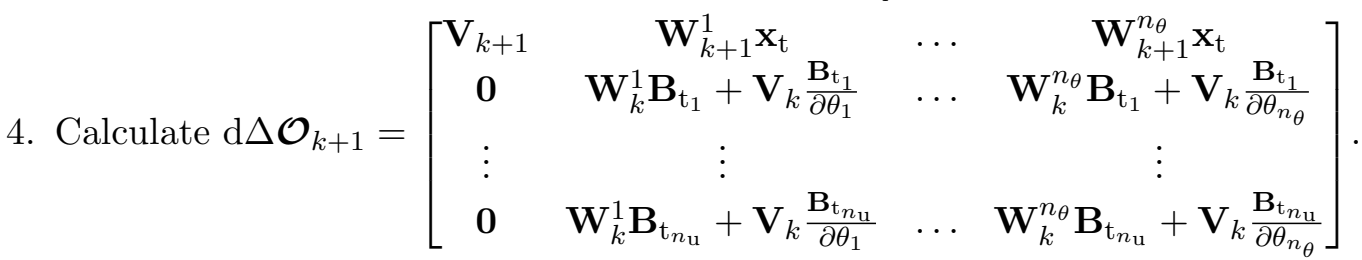

5. Calculate $\mathrm{d} \mathcal{O}_{k+1}=\left[\begin{array}{ll}\mathrm{d} \mathcal{O}_{k}^{\mathrm{T}} & \mathrm{d} \Delta \mathcal{O}_{k+1}^{\mathrm{T}}\end{array}\right]^{\mathrm{T}}$.

6. If $\operatorname{rank}\left(\mathrm{d} \mathcal{O}_{k+1}\right)=\operatorname{rank}\left(\mathrm{d} \mathcal{O}_{k}\right)$, or $\operatorname{rank}\left(\mathrm{d} \mathcal{O}_{k+1}\right)=n_{\mathrm{s}}$, or $k=n_{\mathrm{s}}-2$, end.

7. $k=k+1$ and go to step 3 . 


\subsection{System matrix derivatives}

The application of the ORC algorithm in Section 2.4 requires the derivatives of the system matrices with respect to the unknown parameters $\theta_{q}$. This section provides these derivatives (see also [16]).

Based on Eqs. (7) - (10), the following expressions are derived for the derivatives of the system matrices $\mathbf{A}_{\mathrm{t}}, \mathbf{B}_{\mathrm{t}}, \mathbf{G}_{\mathrm{t}}$, and $\mathbf{J}$ with respect to the parameters $\theta_{q}\left(q=1, \ldots, n_{\theta}\right)$ :

$$
\begin{aligned}
& \frac{\partial \mathbf{A}_{\mathrm{t}}}{\partial \theta_{q}}=\left[\begin{array}{cccc}
\mathbf{0} & \mathbf{0} & \mathbf{0} & \mathbf{0} \\
\mathbf{0} & \mathbf{0} & \mathbf{0} & \mathbf{0} \\
-2 \boldsymbol{\Omega} \frac{\partial \boldsymbol{\Omega}}{\partial \theta_{q}} & \mathbf{0} & -\frac{\partial \mathbf{C}_{\mathrm{m}}}{\partial \theta_{q}} & \mathbf{0} \\
\mathbf{0} & \mathbf{0} & \mathbf{0} & \mathbf{0}
\end{array}\right], \quad \frac{\partial \mathbf{B}_{\mathrm{t}}}{\partial \theta_{q}}=\left[\begin{array}{c}
\mathbf{0} \\
\mathbf{0} \\
\frac{\partial \boldsymbol{\Phi}^{\mathrm{T}}}{\partial \theta_{q}} \mathbf{S}_{\mathrm{u}} \\
\frac{\partial \mathbf{\Phi}_{\mathbf{u}}^{\prime}}{\partial \theta_{q}}
\end{array}\right]
\end{aligned}
$$

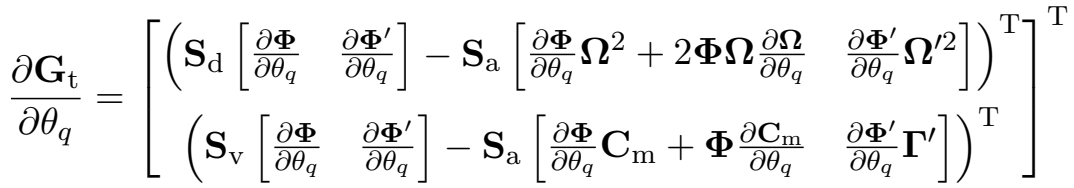

$$
\begin{aligned}
& \frac{\partial \mathbf{J}}{\partial \theta_{q}}=\mathbf{S}_{\mathrm{a}}\left(\frac{\partial \boldsymbol{\Phi}}{\partial \theta_{q}} \boldsymbol{\Phi}^{\mathrm{T}}+\boldsymbol{\Phi} \frac{\partial \boldsymbol{\Phi}^{\mathrm{T}}}{\partial \theta_{q}}\right) \mathbf{S}_{\mathrm{u}}+\mathbf{S}_{\mathrm{a}}\left(\frac{\partial \boldsymbol{\Phi}^{\prime}}{\partial \theta_{q}} \boldsymbol{\Phi}_{\mathrm{u}}^{\prime}+\boldsymbol{\Phi}^{\prime} \frac{\partial \boldsymbol{\Phi}_{\mathrm{u}}^{\prime}}{\partial \theta_{q}}\right)
\end{aligned}
$$

In the derivation of Eqs (25) - (27), it is assumed that the natural frequencies and modal damping ratios assigned to the dummy modes are only determined by the upper frequency limit $\omega_{u}$ considered in the analysis [15] and are therefore independent of the parameters $\theta_{q}$ (see also Section 2.3):

$$
\frac{\partial \omega_{n}^{\prime}}{\partial \theta_{q}}=0, \text { and } \frac{\partial \xi_{n}^{\prime}}{\partial \theta_{q}}=0
$$

Two different cases can be considered. When the unknown parameters $\theta_{q}$ consist of modal parameters, i.e. natural frequencies $\omega_{m}$, components of the mode shape vectors $\phi_{m}$ or the modal damping matrix $\mathbf{C}_{\mathrm{m}}$, the derivatives $\partial \boldsymbol{\Omega} / \partial \theta_{q}, \partial \boldsymbol{\Phi} / \partial \theta_{q}$, and $\partial \mathbf{C}_{\mathrm{m}} / \partial \theta_{q}$ occurring in Eqs. (25-27) can be straightforwardly computed analytically. When, alternatively, the unknown parameters $\theta_{q}$ consist of the underlying physical parameters of the system, i.e. parameters related to mass, stiffness, and/or damping, this is no longer true. In this case, however, one can use the following (exact) expressions that are obtained by applying Nelson's adjoint method [17, 18]:

$$
\begin{aligned}
\frac{\partial \omega_{m}}{\partial \theta_{q}} & =\frac{1}{2 \omega_{m}} \boldsymbol{\phi}_{m}^{\mathrm{T}}\left(\frac{\partial \mathbf{K}}{\partial \theta_{q}}-\omega_{m}^{2} \frac{\partial \mathbf{M}}{\partial \theta_{q}}\right) \boldsymbol{\phi}_{m} \\
\frac{\partial \boldsymbol{\phi}_{m}}{\partial \theta_{q}} & =\left(\mathbf{I}-\boldsymbol{\phi}_{m} \boldsymbol{\phi}_{m}^{\mathrm{T}} \mathbf{M}\right) \mathbf{D}_{m}^{*-1} \mathbf{F}_{m, q}^{*}-\frac{1}{2} \boldsymbol{\phi}_{m} \boldsymbol{\phi}_{m}^{\mathrm{T}} \frac{\partial \mathbf{M}}{\partial \theta_{q}} \boldsymbol{\phi}_{m}
\end{aligned}
$$

where $\mathbf{M} \in \mathbb{R}^{n_{\text {dof }} \times n_{\text {dof }}}$ is the mass matrix. The matrix $\mathbf{D}_{m}^{*} \in \mathbb{R}^{n_{\text {dof }} \times n_{\text {dof }}}$ is used to denote the modified matrix derived from the matrix $\mathbf{D}_{m}$ in Eq. (31) by replacing the elements of the $r$-th column and the $r$-th row by zeros and the $(r, r)$-th element by 1 , where $r$ denotes the element of the mode shape matrix $\phi_{m}$ with the highest absolute value. Similarly, $\mathbf{F}_{m, q}^{*} \in \mathbb{R}^{n_{\text {dof }}}$ is used to denote the modified vector derived from the vector $\mathbf{F}_{m, q}$ in Eq. (32) by replacing the $r$-th element by 0 .

$$
\begin{aligned}
\mathbf{D}_{m} & =\mathbf{K}-\omega_{m}^{2} \mathbf{M} \\
\mathbf{F}_{m, q} & =-\left(\mathbf{I}-\mathbf{M} \boldsymbol{\phi}_{m} \boldsymbol{\phi}_{m}^{\mathrm{T}}\right)\left(\frac{\partial \mathbf{K}}{\partial \theta_{q}}-\omega_{m}^{2} \frac{\partial \mathbf{M}}{\partial \theta_{q}}\right) \boldsymbol{\phi}_{m}
\end{aligned}
$$


The expressions for the modal sensitivities in Eqs. (29) - (32) assume positive natural frequencies $\omega_{m}>0$ and real mode shapes $\phi_{m} \in \mathbb{R}^{n_{\text {dof }}}$ and therefore a positive definite mass matrix $\mathbf{M}>0$ and a positive definite stiffness matrix $\mathbf{K}>0$.

The derivative of the modal damping matrix $\mathbf{C}_{\mathrm{m}}$ with respect to the unknown parameters $\theta_{q}$ is obtained as:

$$
\frac{\partial \mathbf{C}_{\mathrm{m}}}{\partial \theta_{q}}={\frac{\partial \boldsymbol{\Phi}}{\partial \theta_{q}}}^{\mathrm{T}} \mathbf{C}_{\mathrm{v}} \boldsymbol{\Phi}+\boldsymbol{\Phi}^{\mathrm{T}} \frac{\partial \mathbf{C}_{\mathrm{v}}}{\partial \theta_{q}} \boldsymbol{\Phi}+\boldsymbol{\Phi}^{\mathrm{T}} \mathbf{C}_{\mathrm{v}} \frac{\partial \boldsymbol{\Phi}}{\partial \theta_{q}}
$$

In the case of proportional damping, one obtains the following expression:

$$
\frac{\partial \mathbf{C}_{\mathrm{m}}}{\partial \theta_{q}}=\frac{\partial \boldsymbol{\Gamma}}{\partial \theta_{q}}=2 \boldsymbol{\Xi} \frac{\partial \boldsymbol{\Omega}}{\partial \theta_{q}}+2 \boldsymbol{\Omega} \frac{\partial \boldsymbol{\Xi}}{\partial \theta_{q}}
$$

where $\boldsymbol{\Xi} \in \mathbb{R}^{n_{\mathrm{m}} \times n_{\mathrm{m}}}$ is a diagonal matrix containing the modal damping ratios $\xi_{m}$ on its diagonal.

The derivatives of the dummy modes with respect to the parameters $\theta_{q}$ are given by:

$$
\begin{gathered}
\frac{\partial \phi_{\mathrm{u} n}^{\prime}}{\partial \theta_{q}}=\frac{\omega_{n}^{\prime 2}}{2 \phi_{\mathrm{u} n}^{\prime}}\left(\mathbf{S}_{\mathrm{u}_{n}}^{\mathrm{T}} \mathbf{K}^{-1} \frac{\partial \mathbf{K}}{\partial \theta_{q}} \mathbf{K}^{-1} \mathbf{S}_{\mathrm{u}_{n}}+2 \sum_{m=1}^{n_{\mathrm{m}}} \frac{\mathbf{S}_{\mathrm{u}_{n}}^{\mathrm{T}} \boldsymbol{\phi}_{m} \boldsymbol{\phi}_{m}^{\mathrm{T}} \mathbf{S}_{\mathrm{u}_{n}}}{\omega_{m}^{3}} \frac{\partial \omega_{m}}{\partial \theta_{q}} \ldots\right. \\
\left.-\sum_{m=1}^{n_{\mathrm{m}}} \frac{\mathbf{S}_{\mathrm{u}_{n}}^{\mathrm{T}}}{\omega_{m}^{2}}\left(\frac{\partial \boldsymbol{\phi}_{m}}{\partial \theta_{q}} \boldsymbol{\phi}_{m}^{\mathrm{T}}+\boldsymbol{\phi}_{m} \frac{\partial \boldsymbol{\phi}_{m}^{\mathrm{T}}}{\partial \theta_{q}}\right) \mathbf{S}_{\mathrm{u}_{n}}\right)
\end{gathered}
$$

and

$$
\begin{aligned}
\frac{\partial \phi_{\mathrm{d} n}^{\prime}}{\partial \theta_{q}}=\frac{\omega_{n}^{\prime 2}}{\phi_{\mathrm{u} n}^{\prime}}\left(\mathbf{K}^{-1}\right. & \frac{\partial \mathbf{K}}{\partial \theta_{q}} \mathbf{K}^{-1} \mathbf{S}_{\mathrm{u}_{n}}+2 \sum_{m=1}^{n_{\mathrm{m}}} \frac{\boldsymbol{\phi}_{m} \boldsymbol{\phi}_{m}^{\mathrm{T}} \mathbf{S}_{\mathrm{u}_{n}}}{\omega_{m}^{3}} \frac{\partial \omega_{m}}{\partial \theta_{q}} \\
& \left.-\sum_{m=1}^{n_{\mathrm{m}}} \frac{1}{\omega_{m}^{2}}\left(\frac{\partial \boldsymbol{\phi}_{m}}{\partial \theta_{q}} \boldsymbol{\phi}_{m}^{\mathrm{T}}+\boldsymbol{\phi}_{m} \frac{\partial \phi_{m}^{\mathrm{T}}}{\partial \theta_{q}}\right) \mathbf{S}_{\mathrm{u}_{n}}\right)-\frac{\phi_{\mathrm{d} n}^{\prime}}{\phi_{\mathrm{u} n}^{\prime}} \frac{\partial \phi_{\mathrm{u} n}^{\prime}}{\partial \theta_{q}}
\end{aligned}
$$

\subsection{Observability in case of frequency-dependent model accuracy}

As explained in Section 2.2 and illustrated in Example 2.1, the modally reduced order model with quasi-static correction $\mathcal{M}$ is a candidate model which only accurately describes the behavior of the underlying system $\mathcal{S}$ in a limited pre-defined frequency range. This section investigates how such frequency-dependent model accuracy affects the observability test.

Assume that the linear system model $\mathcal{M}$ given in Eqs. (3-4) only provides an adequate representation of the dynamic behavior of a system $\mathcal{S}$ in a limited frequency range $\omega_{1} \leq \omega \leq \omega_{\mathrm{u}}$, with $\omega$ the angular frequency in rad/s. This can be accounted for in the system description by replacing the response $\mathbf{y}$ with the windowed response $\mathbf{y}_{\mathrm{w}}$ obtained after application of a rectangular frequency domain window $H_{\mathrm{w}}(\omega)$ to $\mathbf{y}$, i.e. $\mathbf{y}_{\mathrm{w}}(\omega)=H_{\mathrm{w}}(\omega) \mathbf{y}(\omega)$. Note that $\mathbf{y}_{\mathrm{w}}$ is an accurate representation of the response of $\mathcal{S}$ in the considered frequency range $\omega_{\mathrm{l}} \leq \omega \leq \omega_{\mathrm{u}}$. An example of such a rectangular frequency-domain window $H_{\mathrm{w}}(\omega)$ with its corresponding impulse response $h_{\mathrm{w}}(t)=\mathscr{F}^{-1}\left\{H_{\mathrm{w}}(\omega)\right\}$ is shown in Fig. 3, where $\mathscr{F}^{-1}$ denotes the inverse Fourier transform. The cutoff frequencies of the frequency domain window shown in Fig. 3 have been intentionally chosen so that they correspond to the case in Example 2.1, with $\omega_{\mathrm{l}}=0 \mathrm{rad} / \mathrm{s}$ and $\omega_{\mathrm{u}}=1000 \times 2 \pi \mathrm{rad} / \mathrm{s}$. 

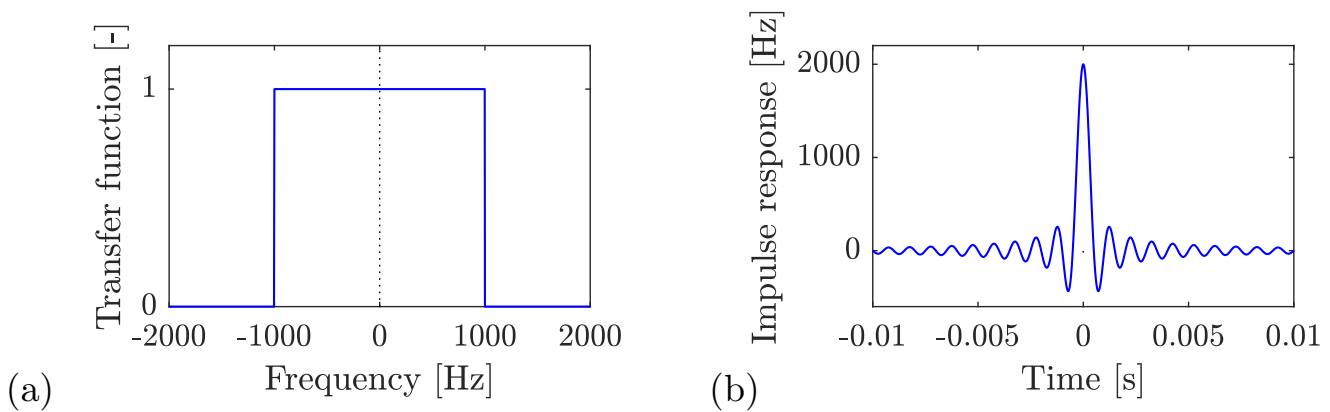

Fig. 3: (a) Transfer function and (b) impulse response of a rectangular frequency domain window with $\omega_{1}=0 \mathrm{rad} / \mathrm{s}$ and $\omega_{\mathrm{u}}=1000 \times 2 \pi \mathrm{rad} / \mathrm{s}$.

The system response $\mathbf{y}_{\mathrm{w}}$ obtained after the application of the rectangular frequency domain window satisfies:

$$
\mathbf{y}_{\mathrm{w}}(t)=\mathbf{y}(t) * h_{\mathrm{w}}(t)
$$

where $*$ denotes the convolution operator. Under the assumption that $\dot{\boldsymbol{\theta}}=\mathbf{0}$, the filtered response $\mathbf{y}_{\mathrm{w}}$ satisfies the following modified system description:

$$
\begin{aligned}
{\left[\begin{array}{c}
\dot{\mathbf{x}}_{\mathrm{t}_{\mathrm{w}}} \\
\dot{\boldsymbol{\theta}}
\end{array}\right] } & =\mathbf{A}(\boldsymbol{\theta})\left[\begin{array}{c}
\mathbf{x}_{\mathrm{t}_{\mathrm{w}}} \\
\boldsymbol{\theta}
\end{array}\right]+\mathbf{B}(\boldsymbol{\theta}) \mathbf{u}_{\mathrm{w}} \\
\mathbf{y}_{\mathrm{w}} & =\mathbf{G}(\boldsymbol{\theta})\left[\begin{array}{c}
\mathbf{x}_{\mathrm{t}_{\mathrm{w}}} \\
\boldsymbol{\theta}
\end{array}\right]+\mathbf{J}(\boldsymbol{\theta}) \mathbf{u}_{\mathrm{w}}
\end{aligned}
$$

where $\mathbf{x}_{\mathrm{t}_{\mathrm{w}}}=\mathbf{x}_{\mathrm{t}} * h_{\mathrm{w}}$, and $\mathbf{u}_{\mathrm{w}}=\mathbf{u} * h_{\mathrm{w}}$. From a practical point of view, only the observability of the windowed states $\mathbf{x}_{\mathrm{t}_{\mathrm{w}}}$ and the parameters $\boldsymbol{\theta}$ from the windowed response $\mathbf{y}_{\mathrm{w}}$ in Eqs. (38-39) is of interest, rather than considering all possible frequencies. From the comparison of Eqs. (3-4) with Eqs. (38-39), however, it appears that the windowing operation does not affect the system matrices $\mathbf{A}, \mathbf{B}, \mathbf{G}$, and $\mathbf{J}$ that appear in the observability test. As a result, if the underlying system is linear, the specific frequency window considered does not intervene in the observability test for a given model $\mathcal{M}$. This conclusion is not only valid for the ORC but holds for any observability test.

\subsection{MROM-observability}

Although modeling errors can never be avoided, it is in most cases realistic to assume - from an observability point of view - that the underlying full order model accurately describes the actual system and therefore generates the data $\mathbf{y}$. Although the modally reduced order model in Eqs. (34) and Eqs. (38-39) is able to reproduce the input-output mapping of the full order model in a very efficient way, as illustrated in Example 2.1, it only provides an approximation of the actual system response and, therefore, of the measurements. It is reasonable to assume that the modally reduced order model (MROM) has been constructed to provide a good approximation of the full order model within the predefined frequency band of interest, as such minimizing the following norm of difference:

$$
\left\|\mathbf{y}_{\mathrm{w}}-\mathbf{y}_{\mathrm{w}}^{\mathrm{M}}(\boldsymbol{\theta})\right\|
$$


where $\mathbf{y}_{\mathrm{w}}$ is the windowed actual system response and $\mathbf{y}_{\mathrm{w}}^{\mathrm{M}}(\boldsymbol{\theta})$ is the windowed response of the modally reduced order model in Eqs. (38-39) with parameters $\boldsymbol{\theta}$ and (windowed) states $\mathbf{x}_{\mathrm{t}_{\mathrm{w}}}$. Note that the parameters $\boldsymbol{\theta}$ may correspond to physical parameters of the underlying full order model as well as modal parameters (Section 2.5). The parameters $\boldsymbol{\theta}^{\star}$ and initial states $\mathbf{x}_{\mathrm{t}_{\mathrm{w}}}^{\star}(0)$ of the reduced order model that minimize the norm in Eq. (40) are in practice what is sought for in an identification procedure. The corresponding response that minimizes this norm is denoted as $\mathbf{y}_{\mathrm{w}}^{\mathrm{M}^{\star}}$.

Now, consider the result of applying the ORC for the reduced order model in Eqs. (38-39). The result would indicate whether, given $\mathbf{y}_{\mathrm{w}}^{\mathrm{M}}$ and $\mathbf{u}_{\mathrm{w}}$, the parameters $\boldsymbol{\theta}$ and states $\mathbf{x}_{\mathrm{t}_{\mathrm{w}}}$ would be observable, i.e. whether they can be distinguished from their immediate neighbors. It should be reminded that the previous observability result refers to a structural property of the system, i.e., it is independent of the exact realizations of $\mathbf{y}_{\mathrm{w}}^{\mathrm{M}}$ and $\mathbf{u}_{\mathrm{w}}$. If the modally reduced order model is deemed to be unobservable, then it will also be unobservable for $\mathbf{y}_{\mathrm{w}}^{\mathrm{M}}=\mathbf{y}_{\mathrm{w}}^{\mathrm{M}^{\star}}$. In such a case, the optimization of (40) will lead to an infinity of solutions, indicating that an identification procedure would not be successful in distinguishing the optimal state and/or parameter values.

Hence, the observability analysis of a candidate modally reduced order model offers useful information about whether or not it is possible to correctly estimate its states and parameters. Since the actual measured signal is $\mathbf{y}$ or $\mathbf{y}_{\mathrm{w}}$, and not $\mathbf{y}_{\mathrm{w}}^{\mathrm{M}}$, as strictly speaking the definition of observability would require, the results of the application of the ORC to a modally reduced order model will be referred to as MROM-observability. Summarizing the previous, if the candidate model is MROM-observable, it is possible to identify its parameters $\boldsymbol{\theta}^{\star}$ and states $\mathbf{x}_{\mathrm{t}_{\mathrm{w}}}^{\star}(0)$, by seeking the minimization of Eq. (40). If, on the contrary, some of the states or parameters are unobservable, there is an infinity of realizations that minimizes the norm in Eq. (40) and the corresponding results of such an identification procedure for those states/parameters would be meaningless.

\subsection{Evaluation of the ORC algorithm}

The calculations in the different steps of the ORC algorithm are generally performed by means of symbolic calculations (for example [2]). In the case of modally reduced order models, however, a full symbolic calculation is very difficult or even impossible. This is due to the fact that symbolic expressions for the natural frequencies $\omega_{m}$ and mode shape vectors $\phi_{m}$, which occur in the expressions for the Jacobian matrix $\mathrm{d} \mathcal{O}_{k+1}$, can no longer be obtained for structures with a large number of degrees of freedom. An alternative way to accurately evaluate the different steps of the ORC algorithm is therefore developed. The different steps are outlined next.

1. Choose representative values $\boldsymbol{\theta}^{\star}$ for the parameters $\boldsymbol{\theta}$ and compose the mass matrix $\mathbf{M}\left(\boldsymbol{\theta}^{\star}\right)$, the viscous damping matrix $\mathbf{C}_{\mathrm{v}}\left(\boldsymbol{\theta}^{\star}\right)$ and the stiffness matrix $\mathbf{K}\left(\boldsymbol{\theta}^{\star}\right)$.

2. Solve the generalized eigenvalue problem $\mathbf{K} \phi_{m}=\omega_{m}^{2} \mathbf{M} \phi_{m}$ to obtain the natural frequencies $\omega_{m}$ and the mode shape vectors $\phi_{m}$ with standard double precision (MATLAB function 'eig.m' or 'eigs.m'). Note that MATLAB function 'eigs.m' only computes a pre-defined number of approximations to eigenvectors. These are not necessarily accurate approximations. On the other hand, MATLAB function 'eig.m' computes all $\left(n_{\text {dof }}\right)$ eigenvectors, which is computationally much more demanding, especially for large problems. The obtained eigenvectors are generally more accurate than those obtained from 'eigs.m'. In both cases, however, a refinement of the obtained natural frequencies and mode shapes is compulsory for our purposes. 
3. Evaluate the mass matrix $\mathbf{M}$, the viscous damping matrix $\mathbf{C}_{\mathrm{v}}$, the stiffness matrix $\mathbf{K}$, the natural frequencies $\omega_{m}$, and the mode shape vectors $\phi_{m}$ with increased 128 decimal digit precision. The same precision is adopted in the following steps, including the actual evaluation of the ORC. For problems with a small number of degrees of freedom $\left(n_{\text {dof }}<500\right)$, this high-precision evaluation can be performed using variable-precision floating-point arithmetic (VPA, MATLAB function 'vpa.m'). For larger problems, such as the one considered in Section 3.2, the Advanpix Multiprecision Computing Toolbox for MATLAB [19] can be adopted, which enables a significant speed-up of the calculations with respect to VPA. This toolbox is used here for all examples considered in Section 3.

4. Perform a series of Rayleigh quotient iterations [20] to obtain increasingly accurate estimates of the natural frequencies $\omega_{m}$ and the corresponding mode shape vectors $\phi_{m}$.

a. Initialization: $l=0, \epsilon^{*}=10^{-112}, \lambda_{m, 0}=\omega_{m}^{2}, \phi_{m, 0}=\phi_{m} /\left\|\phi_{m}\right\|_{2}$.

b. $l=1+1$.

c. Calculate $\boldsymbol{\psi}=\left(\mathbf{K}-\lambda_{m, l-1} \mathbf{M}\right)^{-1}\left(\mathbf{M} \phi_{m, l-1}\right)$.

d. Calculate $\boldsymbol{\phi}_{m, l}=\boldsymbol{\psi} /\|\boldsymbol{\psi}\|_{2}, \lambda_{m, l}=\left(\boldsymbol{\phi}_{m, l}^{\mathrm{T}} \mathbf{M}^{-1}\right)\left(\mathbf{K} \boldsymbol{\phi}_{m, l}\right) /\left(\boldsymbol{\phi}_{m, l}^{\mathrm{T}} \boldsymbol{\phi}_{m, l}\right)$.

e. Calculate $\epsilon_{l}=\left\|\mathbf{K} \phi_{m, l}-\lambda_{m, l} \mathbf{M} \phi_{m, l}\right\|_{2}$.

f. If $\epsilon_{l} \geq \epsilon^{*}$, go to step b.

g. Post-processing: $\phi_{m}=\phi_{m, l} / \sqrt{\phi_{m, l}^{\mathrm{T}} \mathbf{M} \phi_{m, l}}$ (mass normalization), $\omega_{m}=\sqrt{\lambda_{m, l}}$.

The iteration scheme is terminated when the error $\epsilon_{l}$ obtained for the current estimate of the squared natural frequency $\lambda_{m, l}$ and the corresponding estimate of the mode shape $\phi_{m, l}$ is smaller than a predefined threshold $\epsilon^{*}$. The precision $\epsilon^{*}$ is related to the decimal digit precision adopted in the calculations as follows. If we want to achieve a precision of 112 digits $\left(\epsilon^{*}=10^{-112}\right)$, we compute with 128 digits $(112+16$, where 16 links to the machine precision). If a higher precision is desired, the rule of thumb will be to add 16 additional digits to the required precision. The Rayleigh quotient iteration scheme has a cubic convergence of the eigenvalues $\lambda_{m, l}$. As a result, only a few steps are required to obtain the predefined accuracy. Note that omitting the refinement of the modal parameters by means of Rayleigh quotient iterations may lead to inaccurate results of the observability test. This is further illustrated in Section 3.1.

5. Calculate the matrix derivatives $\frac{\partial \mathbf{M}}{\partial \theta_{q}}, \frac{\partial \mathbf{K}}{\partial \theta_{q}}$ and $\frac{\partial \mathbf{C}_{\mathbf{v}}}{\partial \theta_{q}}$ or, in the case of proportional damping $\frac{\partial \mathbf{M}}{\partial \theta_{q}}, \frac{\partial \mathbf{K}}{\partial \theta_{q}}$ and $\frac{\partial \boldsymbol{\Xi}}{\partial \theta_{q}}$. These matrix derivatives can in most cases be calculated analytically, or alternatively using finite differences or complex step differentiation.

6. Calculate the matrix derivatives $\frac{\partial \mathbf{A}_{t}}{\partial \theta_{q}}, \frac{\partial \mathbf{B}_{t}}{\partial \theta_{q}}$, and $\frac{\partial \mathbf{G}_{\mathbf{t}}}{\partial \theta_{q}}$ using Eqs. (25-26) and (29-36).

One is designated to choosing parameter values $\boldsymbol{\theta}^{\star}$ in a realistic operating range, which requires some a priori knowledge on the structure. If the system is found to be observable for a given set of parameter values, this ensures observability in a closed neighborhood of the selected parameter values $\boldsymbol{\theta}^{\star}$. Similarly, one is designated to choosing a realization of the state vector $\mathbf{x}_{\mathrm{t}}$ for the evaluation of the ORC algorithm. It was found that this choice generally does not affect the outcome of the observability test, as long as (unrealistic) very high and low values are avoided, 
which may lead to numerical problems. For the examples provided in Section 3, random values are drawn from a normal distribution with zero mean and unit standard deviation.

For a given parameter and state realization, one cannot ensure that the chosen parameter and state values do not coincide with a singular point, i.e. a point at which the rank is smaller than for any other general realization. It is highly unlikely, however, that a randomly chosen realization would correspond to such a singular point. Hence, one can consider that the calculated observability property for a single realization is accurate with a high probability of confidence, as for example described by Sedoglavic in [8]. In any case, if the system turns out to be unobservable, one can always try different parameter and state values and repeat the investigation to avoid singularities.

\section{Examples}

\subsection{A linear $4 D O F$ system}

In this example, the observability of a simple shear building with four storeys is examined. The model and its corresponding mass, damping, and stiffness matrices are shown in Fig. 4. The displacement, velocity, and acceleration of storey $j$ are denoted as $x_{j}, \dot{x}_{j}$, and $\ddot{x}_{j}$, respectively. Every floor $j(j=1, \ldots, 4)$ has a mass $m_{j}$ and is connected to floor $j-1$ by linear springs with stiffness $k_{j}$ and linear dashpots with damping constant $c_{j}$. A known force $u$ is applied at the fourth floor. The parameters to be identified are the spring constants $k_{1}, k_{2}, k_{3}$, and $k_{4}$, and the damping constants $c_{1}, c_{2}, c_{3}$, and $c_{4}$. The masses $m_{1}, m_{2}, m_{3}$, and $m_{4}$ are assumed known and equal $2 \mathrm{~kg}$. The measurements consist of the displacement at the second and the top floor, $x_{2}$ and $x_{4}$, respectively.

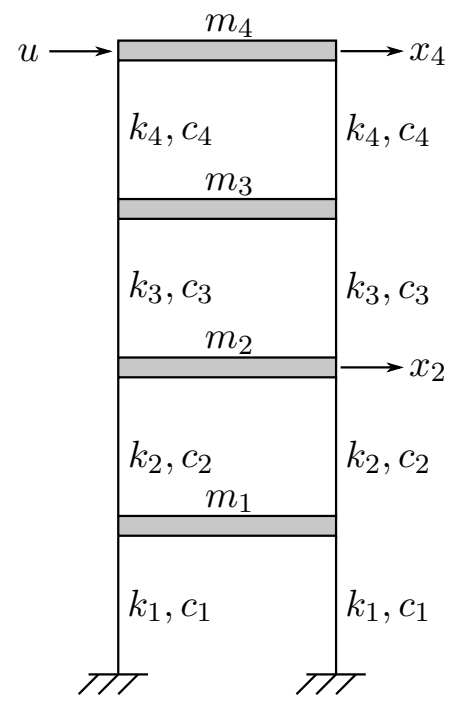

$$
\begin{aligned}
\mathbf{M} & =\left[\begin{array}{cccc}
m_{1} & 0 & 0 & 0 \\
0 & m_{2} & 0 & 0 \\
0 & 0 & m_{3} & 0 \\
0 & 0 & 0 & m_{4}
\end{array}\right] \\
\mathbf{C}_{\mathrm{v}} & =\left[\begin{array}{cccc}
2 c_{1}+2 c_{2} & -2 c_{2} & 0 & 0 \\
-2 c_{2} & 2 c_{2}+2 c_{3} & -2 c_{3} & 0 \\
0 & -2 c_{3} & 2 c_{3}+2 c_{4} & -2 c_{4} \\
0 & 0 & -2 c_{4} & 2 c_{4}
\end{array}\right] \\
\mathbf{K} & =\left[\begin{array}{cccc}
2 k_{1}+2 k_{2} & -2 k_{2} & 0 & 0 \\
-2 k_{2} & 2 k_{2}+2 k_{3} & -2 k_{3} & 0 \\
0 & -2 k_{3} & 2 k_{3}+2 k_{4} & -2 k_{4} \\
0 & 0 & -2 k_{4} & 2 k_{4}
\end{array}\right]
\end{aligned}
$$

Fig. 4: 4DOF shear building model considered in Example 3.1 and its mass, damping, and stiffness matrices.

First, as a reference case, the observability of the full order model expressed in physical coordinates is investigated. The state vector $\mathbf{x}_{\mathrm{t}}$ consists of the displacements $x_{j}$ and velocities $\dot{x}_{j}$ of the 
four floors $(j=1, \ldots, 4)$. The system matrices $\mathbf{A}_{\mathrm{t}}, \mathbf{B}_{\mathrm{t}}, \mathbf{G}_{\mathrm{t}}$, and $\mathbf{J}$ are given by:

$$
\begin{aligned}
\mathbf{A}_{\mathrm{t}} & =\left[\begin{array}{cc}
\mathbf{0} & \mathbf{I} \\
-\mathbf{M}^{-1} \mathbf{K} & -\mathbf{M}^{-1} \mathbf{C}_{\mathrm{v}}
\end{array}\right], \quad \mathbf{B}_{\mathrm{t}}=\left[\begin{array}{c}
\mathbf{0} \\
\mathbf{M}^{-1} \mathbf{S}_{\mathrm{u}}
\end{array}\right] \\
\mathbf{G}_{\mathrm{t}} & =\left[\begin{array}{ll}
\mathbf{S}_{\mathrm{d}}-\mathbf{S}_{\mathrm{a}} \mathbf{M}^{-1} \mathbf{K} & \mathbf{S}_{\mathrm{v}}-\mathbf{S}_{\mathrm{a}} \mathbf{M}^{-1} \mathbf{C}_{\mathrm{v}}
\end{array}\right], \quad \mathbf{J}=\mathbf{S}_{\mathrm{a}} \mathbf{M}^{-1} \mathbf{S}_{\mathrm{u}}
\end{aligned}
$$

Applying the ORC in its reduced form, presented in Section 2.4, reveals that all dynamic states $\mathbf{x}_{\mathrm{t}}$ and parameters of the model $\boldsymbol{\theta}$ are observable (see also Table 1).

\begin{tabular}{llll}
\hline Case & Description & Observable states & Unobservable states \\
\hline 1 & Full order model, & $x_{1}, x_{2}, x_{3}, x_{4}, \dot{x}_{1}, \dot{x}_{2}, \dot{x}_{3}, \dot{x}_{4}$, & - \\
& physical coordinates, & $k_{1}, k_{2}, k_{3}, k_{4}, c_{1}, c_{2}, c_{3}, c_{4}$ & \\
& physical parameters & & \\
\hline 2 & Reduced order model, & $z_{1}, z_{2}, z_{1}^{\prime}, \dot{z}_{1}, \dot{z}_{2}, \dot{z}_{1}^{\prime}$, & $c_{1}, c_{2}, c_{3}, c_{4}$ \\
& general viscous damping & $k_{1}, k_{2}, k_{3}, k_{4}$ & \\
\hline 3 & Reduced order model, & $z_{1}, z_{2}, z_{1}^{\prime}, \dot{z}_{1}, \dot{z}_{2}, \dot{z}_{1}^{\prime}$, & - \\
& proportional damping & $k_{1}, k_{2}, k_{3}, k_{4}, \xi_{1}, \xi_{2}$ & \\
\hline
\end{tabular}

Table 1: Overview of the partially observable and unobservable states for the different cases investigated by means of the ORC for the 4 DOF system.

Second, we consider the reduced order model that accurately describes the system in the frequency range from 0 to $10 \mathrm{~Hz}$. The prior estimate $k_{j}=3000 \mathrm{~N} / \mathrm{m}$ and $c_{j}=3 \mathrm{Ns} / \mathrm{m}, j=1, \ldots, 4$, is considered in the observability evaluation. For the provided parameter values, the four natural frequencies of the structure are $3.03 \mathrm{~Hz}, 8.72 \mathrm{~Hz}, 13.36 \mathrm{~Hz}$, and $16.38 \mathrm{~Hz}$.

A reduced order model is obtained by retaining the dynamic contribution of the first two structural modes. The quasi-static contribution of the two higher modes is accounted for by means of a single dummy mode $\left(n_{\mathrm{u}}=1\right)$. The natural frequency and modal damping ratio of the dummy mode are assigned values of $15 \mathrm{~Hz}$ and $0.5 \%$, respectively, as to obtain a nearly constant (i.e. quasistatic) contribution within the frequency range of interest. In this case, the state vector $\mathbf{x}_{\mathrm{t}}$ consists of the modal displacements and velocities corresponding to the first two structural modes $\left(z_{1}, z_{2}\right.$, $\dot{z}_{1}$, and $\left.\dot{z}_{2}\right)$ and the modal displacement and velocity corresponding to the dummy mode $\left(z_{1}^{\prime}\right.$ and $\left.\dot{z}_{1}^{\prime}\right)$, see Eq. (6). Fig. 5 compares the transfer function of the full order model $H_{\mathrm{yu}}(\omega)$ to the transfer function of the modally reduced order model with dummy mode correction $H_{\mathrm{yu}}^{\prime \prime}(\omega)$, considering output $x_{4}$ and input $u$. As for the case of the simply supported beam in Example 2.1, a very good overall agreement between the two transfer functions is obtained in the frequency range from 0 to $10 \mathrm{~Hz}$, whereas the two transfer functions are clearly different for frequencies above $10 \mathrm{~Hz}$.

In addition to the assumed parameter values, random values are assigned to the state vector $\mathbf{x}_{\mathrm{t}}$ (see also Section 2.8). Application of the methodology proposed in Section 2.2 for the reduced order model with quasi-static correction reveals that the dynamic states $\mathbf{x}_{\mathrm{t}}$, consisting of the modal displacements and velocities, are MROM-observable. The stiffness constants $k_{1}, k_{2}, k_{3}$, and $k_{4}$ are identifiable. The damping constants $c_{1}, c_{2}, c_{3}$, and $c_{4}$, however, are not MROM-identifiable and hence cannot be independently determined from the data. This is because the two highest modes are assumed to only contribute to the response in a quasi-static way. As such, their contribution only contains information on the stiffness of the structure, not on its damping or mass. 


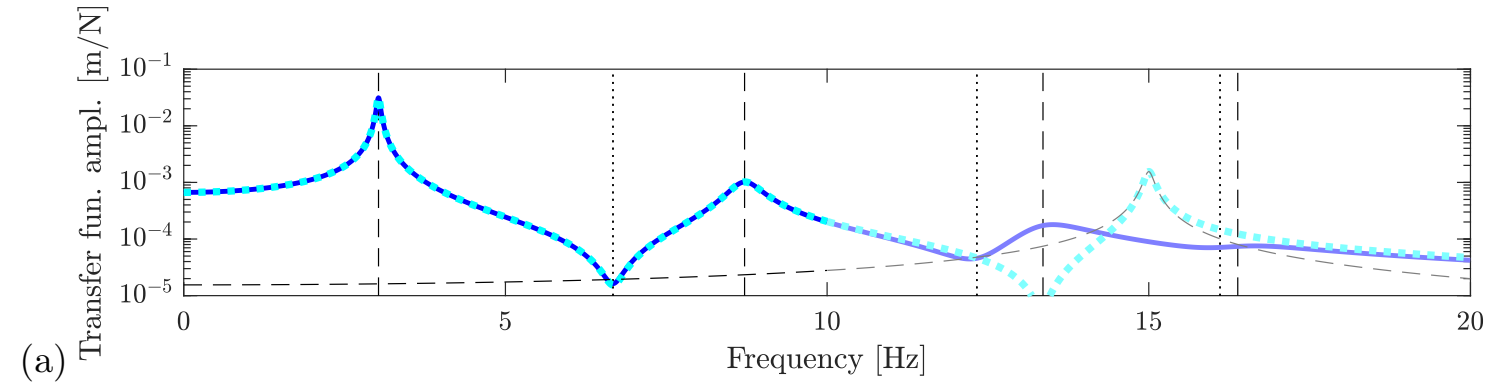

(a)

Frequency $[\mathrm{Hz}]$

(b)

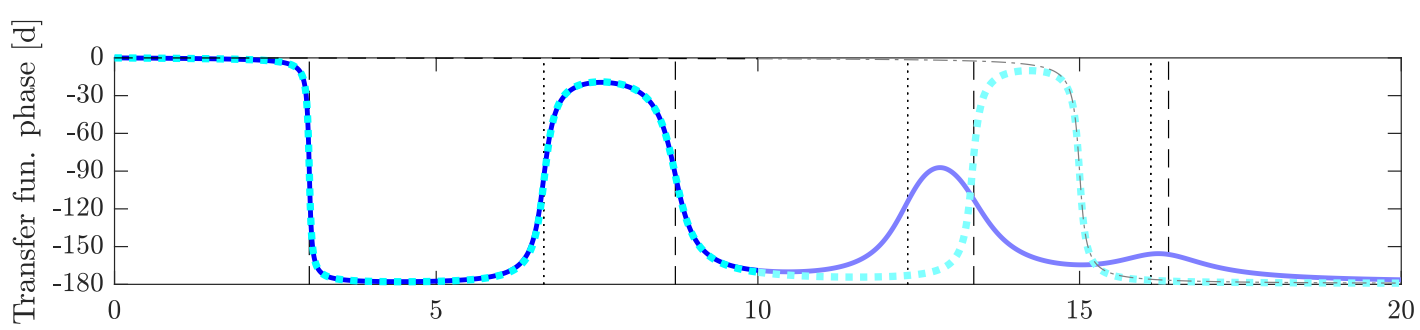

Fig. 5: (a) Amplitude and (b) phase of transfer functions $H_{\mathrm{yu}}(\omega)$ (4 modes, blue solid line) and $H_{\mathrm{yu}}^{\prime \prime}(\omega)(2$ modes with dummy mode correction, cyan dotted line) that relate displacement $x_{4}$ to force $u$. The contribution of the dummy mode is indicated by a black dashed line. The undamped natural frequencies are indicated by vertical dashed lines, the antiresonance frequencies of the full order model by vertical dotted lines.

It is found that the system becomes MROM-observable under the assumption of proportional damping, i.e. by using Eq. (34) instead of Eq. (33) to compute the modal sensitivity $\frac{\partial \mathbf{C}_{m}}{\partial \theta_{q}}$. In this case, the modal damping ratios $\xi_{1}$ and $\xi_{2}$ corresponding to modes 1 and 2 are identifiable, but not the individual damping constants $c_{1}, c_{2}, c_{3}$, and $c_{4}$.

Fig. 6 shows the singular value spectrum of the observability matrix $\mathrm{d} \mathcal{O}_{k+1}$ for case 2 , with $k=4$, obtained using the numerical evaluation procedure discussed in Section 2.8, where a 128 digit precision is adopted in combination with Rayleigh quotient iterations. A comparison is made with the singular value spectrum of the observability matrix obtained using a numerical evaluation of the ORC in double precision and without refinement of the natural frequencies and mode shapes by means of Rayleigh quotient iterations. A clear difference is observed in the smallest singular value with index 14 . For the evaluation in high precision, a clear gap in the singular value spectrum is observed between singular values 13 and 14, leading to numerical rank 13 .

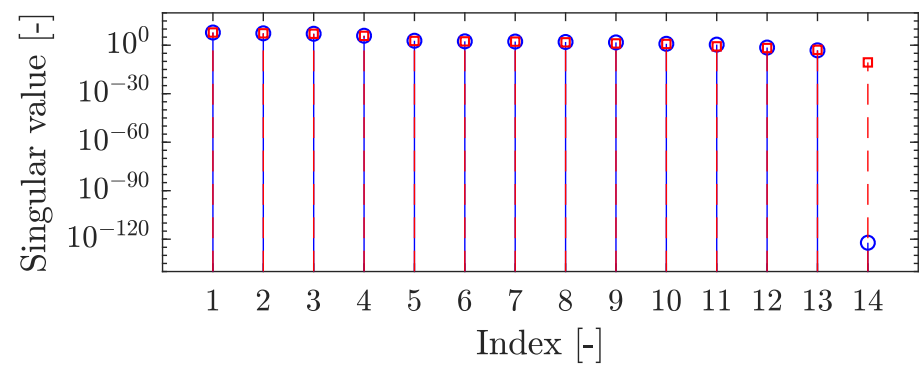

Fig. 6: Singular value spectrum of the observability matrix $\mathrm{d} \mathcal{O}_{5}$ for case 2, obtained using the numerical evaluation procedure discussed in Section 2.8 (blue circles) and obtained using a numerical evaluation in double precision (red squares). 
For the evaluation in double precision, the difference between singular values 13 and 14 is much less pronounced. The absence of a clear gap is due to the accumulation of numerical errors when adopting double precision in all steps, leading to a pollution of the singular value spectrum and therefore an inaccurate estimation of the observability rank. It is therefore advised to always adopt the numerical procedure outlined in Section 2.8 in the evaluation of the ORC.

\subsection{A footbridge in Wetteren, Belgium}

In the second example, we consider the observability for the case of joint state and parameter estimation on a steel footbridge in Wetteren, Belgium, shown in Fig. 7a. This bridge was previously the test subject for in situ validation experiments for operational modal analysis (OMA) in [21] and response estimation by means of joint input-state estimation in [22]. The footbridge crosses the E40 highway between Brussels and Ghent in Wetteren and has a short span with a length of $30.3 \mathrm{~m}$ and a longer bowstring span with a length of $75.2 \mathrm{~m}$.

(a)

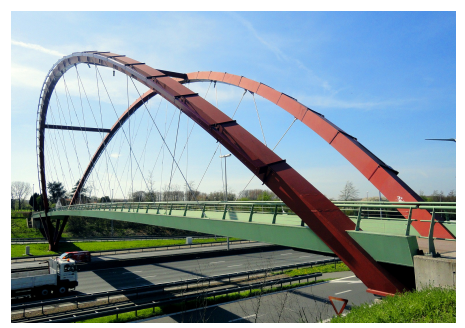

(b)

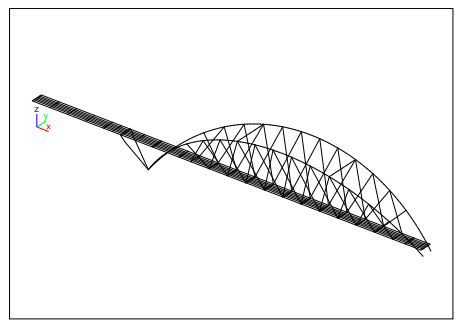

(c)

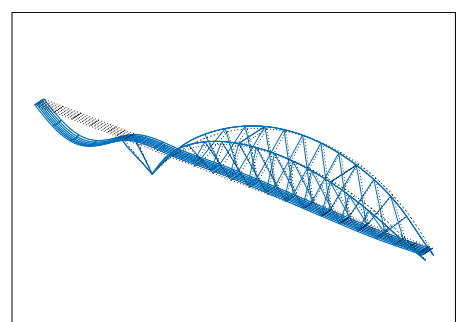

Fig. 7: (a) The footbrigde in Wetteren, Belgium, (b) 3D view of the FE model developed in StaBIL, and (c) 3D view of the first calculated vertical bending mode of the side $\operatorname{span}\left(f_{j}=2.76 \mathrm{~Hz}\right)$.

A finite element model of the footbridge has been developed using the MATLAB toolbox StaBIL [23]. The longitudinal and transverse girders of the bridge deck, the arches, and the support legs at the intersection between the main span and the side span are modeled using beam elements. The cables connecting the arches with the bridge deck are modeled using truss elements. For the steel plate and the asphalt layer on the bridge deck, the added mass is accounted for by increasing the density of the longitudinal bridge deck girders. The added stiffness of these components is not accounted for. The resulting model has a total of 638 nodes, 587 elements, and 2314 degrees of freedom. Figs. 7b and 7c show respectively a three-dimensional view of the FE model and the first calculated vertical bending mode of the side span. The calculated natural frequency of this mode equals $2.76 \mathrm{~Hz}$ and is lower than the value of $3.73 \mathrm{~Hz}$ which was experimentally identified [21]. This difference may potentially be attributed to an underestimation of the stiffness of the bridge deck.

In the remainder of this example, it is investigated to what extent a modally reduced order model of the footbridge can be used in combination with strain measurements on the bridge deck to estimate the system states (modal displacements and velocities) and different uncertain modal and/or physical model parameters. Consider the case where a single person is (slowly) jumping up and down near the center of the side span (Fig. 8). This causes a vertical dynamic load $u$ which is typically contained within the frequency range between 0 and $5 \mathrm{~Hz}$ [24]. For this example, this is the frequency range of interest. We consider the case where the vertical jumping load is measured, for example using a force plate. The measured response $\mathbf{y}$ consists of six so-called distributed 
macro-strains $\epsilon_{1}, \epsilon_{2} \ldots, \epsilon_{6}$ along the neutral axis of the main bridge girders (Fig. 8). These macrostrains consist of the relative longitudinal displacement between two transverse bridge girders and could for example be measured using fiber optic Bragg grating (FBG) sensors [25].

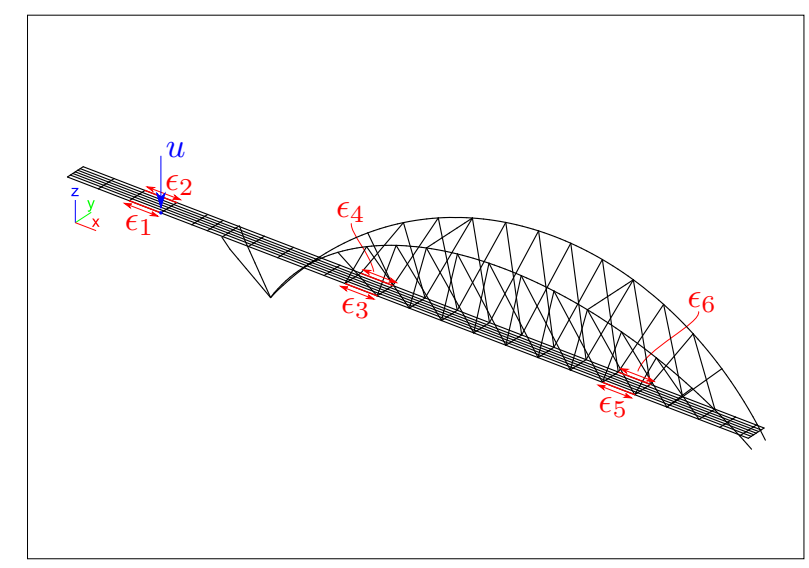

Fig. 8: Overview of the measurement setup for the Wetteren footbridge, with an indication of the applied (known) force $u$ and the macro-strain measurements $\epsilon_{j}$.

As opposed to the previous example in Section 3.1, the default ORC [2] cannot be successfully applied to the full order model of the footbridge with 2314 DOFs, due to limitations in the computer memory. While recent more efficient implementations of the ORC can be applied directly to the full order state-space model [14], they would not be able to offer any information related to the modal properties of the system. As such, their results would not be relevant to the MROM observability, which is of interest for the actual system identification. In this case, we study the observability of a modally reduced order model which accurately describes the dynamic behavior of the full order model in the frequency range of interest, i.e. 0 to $5 \mathrm{~Hz}$. The model under consideration includes the full dynamic contribution of the 13 modes for which the preliminary model predicts a natural frequency below $5 \mathrm{~Hz}$ plus 1 additional mode with a predicted natural frequency of $5.29 \mathrm{~Hz}$ that has a significant dynamic contribution below $5 \mathrm{~Hz}$ (see Fig. 9). For this mode, which corresponds to torsion of the side span, including the quasi-static contribution rather than the full dynamic contribution is insufficient for an accurate representation of the dynamic behavior between 0 and $5 \mathrm{~Hz}$. The quasi-static contribution of the remaining (out-of-band) modes is accounted for by means of a single dummy mode with a natural frequency of $15 \mathrm{~Hz}$ and a modal damping ratio of 0.5\%. As shown in Fig. 9, the quasi-static correction in this case is mainly important in the low frequency range below $2 \mathrm{~Hz}$.

The state vector of the modally reduced order model $\mathbf{x}_{\mathrm{t}}$ consists of the modal displacements and velocities corresponding to the 14 modes that were previously considered $\left(z_{m}\right.$ and $\dot{z}_{m}, m=$ $1, \ldots, 14)$ and the modal displacement and velocity corresponding to the dummy mode $\left(z_{1}^{\prime}\right.$ and $\left.\dot{z}_{1}^{\prime}\right)$. Six different cases are considered in the MROM-observability test, where each case is different in terms of the parameters to be identified and/or the presence of measured and hence known inputs. The results are summarized in Table 2 and are discussed in the remainder of this section.

In the first case, the parameters to be identified consist of the modal parameters, i.e. the natural frequencies $\omega_{m}$, the modal damping ratios $\xi_{m}$, the six macro-strain mode shape components $\phi_{m, \epsilon_{s}}$ ( $m=1, \ldots, 14, s=1, \ldots, 6)$, the vertical displacement mode shape component at the input location $\phi_{m, \mathrm{u}}(m=1, \ldots, 14)$, and the corresponding dummy strain and displacement mode shape 


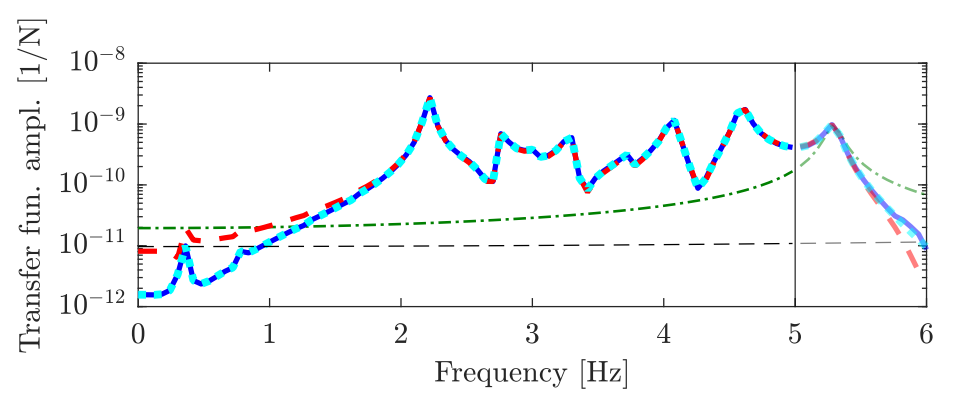

Fig. 9: Amplitude of the transfer functions $H_{\mathrm{yu}}(\omega)$ (full order model, blue solid line), $H_{\mathrm{yu}}^{\prime}(\omega)$ (MROM without quasi-static correction, red dashed line), and $H_{\mathrm{yu}}^{\prime \prime}(\omega)$ (MROM with dummy mode correction, cyan dotted line) that relate macro-strain $\epsilon_{1}$ to force $u$. The contribution of the torsional mode of the side span $\left(f_{j}=5.29 \mathrm{~Hz}\right)$ is indicated by a green dash-dotted line. The contribution of the dummy mode is indicated by a black dashed line.

components $\phi_{\epsilon_{s} 1}^{\prime}(j=1, \ldots, 6)$ and $\phi_{\mathrm{u} 1}^{\prime}$. The total number of unknown parameters equals 133 . The system identification is performed for a known input $u$. Application of the ORC reveals that only the natural frequencies $\omega_{m}$ and modal damping ratios $\xi_{m}$ are MROM-observable. The mode shape components and the corresponding modal displacements and velocities cannot be independently observed from the data. This is due to the fact that the so-called driving point response is not measured, i.e. the response (displacement, velocity or acceleration) at the location and along the direction of the applied input $u$. In such a case, the mode shapes can only be determined up to an arbitrary scaling factor [26]. This is verified in case 2, where the re-scaled macro-strain mode shape components $\phi_{m, \epsilon_{s}} / \phi_{m, \mathrm{u}}$ and $\phi_{\epsilon_{s} 1}^{\prime} / \phi_{\mathrm{u} 1}^{\prime}(m=1, \ldots, 14, s=1, \ldots, 6)$ are identified, instead of the separate (mass-normalized) mode shape components $\phi_{m, \epsilon_{s}}, \phi_{m, \mathrm{u}}, \phi_{\epsilon_{s} 1}^{\prime}$ and $\phi_{\mathrm{u} 1}^{\prime}$. Note that this corresponds to the case where one would assume $\phi_{m, \mathrm{u}}=\phi_{\mathrm{u} 1}^{\prime}=1(m=1, \ldots, 14)$ and successively adopt system identification to determine $\phi_{m, \epsilon_{s}}$ and $\phi_{\epsilon_{s} 1}^{\prime}$. In this case, the number of unknown parameters equals 118. Application of the ORC reveals that all (modal) parameters and dynamic system states are indeed observable.

In the third case, it is investigated to what extent the modal parameters of the system can be identified from a so-called output-only free decay analysis, i.e. when the response is measured in absence of excitation $(u=0)$. If no excitation is present, it is reasonable to assume that the dummy mode, which accounts for the quasi-static contribution of the out-of-band modes, does not contribute to the response, i.e. $z_{1}^{\prime}=\dot{z}_{1}^{\prime}=0$. As such, the system model only includes the dynamic contribution of the 14 modes that were previously considered. The parameters to be identified consist of the natural frequencies $\omega_{m}$, the modal damping ratios $\xi_{m}$, and the six macrostrain mode shape components $\phi_{m, \epsilon_{s}}(m=1, \ldots, 14, s=1, \ldots, 6)$. The total number of unknown parameters equals 112. Similar to case 1, application of the ORC reveals that only the natural frequencies $\omega_{m}$ and modal damping ratios $\xi_{m}$ are MROM-observable, whereas the mode shape components and the corresponding modal displacements and velocities cannot be independently observed. This is due to the fact that an absolute scaling of the mode shapes is not possible in an output-only setting. This is verified in case 4 , where the re-scaled macro-strain mode shape components $\phi_{m, \epsilon_{s}} / \phi_{m, \epsilon_{1}}(m=1, \ldots, 14, s=2, \ldots, 6)$ are identified, instead of the separate (massnormalized) mode shape components $\phi_{m, \epsilon_{s}}$. In this case, the number of unknown parameters is reduced to 98 . As expected, the application of the ORC reveals that all (modal) parameters and dynamic system states are observable. 


\begin{tabular}{|c|c|c|c|}
\hline Case & Description & Observable states & Unobservable states \\
\hline 1 & $\begin{array}{l}\text { Unknown modal parameters, } \\
\text { absolute mode scaling, } \\
\text { known input } u\end{array}$ & $\omega_{m}, \xi_{m}(m=1, \ldots, 14)$ & $\begin{array}{l}\phi_{m, \epsilon_{s}}, \phi_{m, \mathrm{u}}, \phi_{\epsilon_{s} 1}^{\prime}, \phi_{\mathrm{u} 1}^{\prime}, \\
z_{m}, \dot{z}_{m}, z_{1}^{\prime}, \dot{z}_{1}^{\prime} \\
(m=1, \ldots, 14, s=1, \ldots, 6)\end{array}$ \\
\hline 2 & $\begin{array}{l}\text { Unknown modal parameters, } \\
\text { mode scaling assumed known, } \\
\text { known input } u\end{array}$ & $\begin{array}{l}\omega_{m}, \xi_{m}, \phi_{m, \epsilon_{s}} / \phi_{m, \mathrm{u}}, \phi_{\epsilon_{s} 1}^{\prime} / \phi_{\mathrm{u} 1}^{\prime} \\
z_{m}, \dot{z}_{m}, z_{1}^{\prime}, \dot{z}_{1}^{\prime} \\
(m=1, \ldots, 14, s=1, \ldots, 6)\end{array}$ & - \\
\hline 3 & $\begin{array}{l}\text { Unknown modal parameters, } \\
\text { absolute mode scaling, } \\
\text { no known inputs }(u=0)\end{array}$ & $\omega_{m}, \xi_{m}(m=1, \ldots, 4)$ & $\begin{array}{l}\phi_{m, \epsilon_{s}}, z_{m}, \dot{z}_{m} \\
(m=1, \ldots, 14, s=1, \ldots, 6)\end{array}$ \\
\hline 4 & $\begin{array}{l}\text { Unknown modal parameters, } \\
\text { mode scaling assumed known, } \\
\text { no known inputs }(u=0)\end{array}$ & $\begin{array}{l}\omega_{m}, \xi_{m}, \phi_{m, \epsilon_{s}} / \phi_{m, \epsilon_{1}} \\
z_{m}, \dot{z}_{m} \\
(m=1, \ldots, 14, s=2, \ldots, 6)\end{array}$ & - \\
\hline 5 & $\begin{array}{l}\text { Unknown parameters } E_{\mathrm{b}}, \rho_{\mathrm{b}}, \\
\xi_{m}(m=1, \ldots, 14) \\
\text { known input } u\end{array}$ & $\begin{array}{l}E_{\mathrm{b}}, \rho_{\mathrm{b}}, \xi_{m}, z_{m}, \dot{z}_{m}, z_{1}^{\prime}, \dot{z}_{1}^{\prime} \\
(m=1, \ldots, 14)\end{array}$ & - \\
\hline 6 & $\begin{array}{l}\text { Unknown parameters } E_{\mathrm{b}}, \rho_{\mathrm{b}}, \\
\xi_{m}(m=1, \ldots, 14) \\
\text { no known inputs }(u=0)\end{array}$ & $\begin{array}{l}E_{\mathrm{b}}, \rho_{\mathrm{b}}, \xi_{m}, z_{m}, \dot{z}_{m} \\
(m=1, \ldots, 14)\end{array}$ & - \\
\hline
\end{tabular}

Table 2: Overview of the partially observable and unobservable states for the different cases investigated by means of the ORC for the Wetteren footbridge.

In the final two cases 5 and 6 , the parameters to be identified consist of the Young's modulus $E_{\mathrm{b}}$ and the density $\rho_{\mathrm{b}}$ of the longitudinal bridge deck girders, as well as the modal damping ratios $\xi_{m}(m=1, \ldots, 14)$. It is assumed that the remaining material and geometrical properties of the structure are known. For a given value of $E_{\mathrm{b}}$ and $\rho_{\mathrm{b}}$, the modal properties $\omega_{m}, \phi_{m, \epsilon_{s}}, \phi_{m, \mathrm{u}}, \phi_{\epsilon_{s} 1}^{\prime}$ and $\phi_{\mathrm{u} 1}^{\prime}$, which intervene in the system description, can be determined. These modal properties are therefore not considered as additional unknown parameters. The matrix derivatives $\partial \mathbf{K} / \partial E_{\mathrm{b}}$ and $\partial \mathbf{M} / \partial \rho_{\mathrm{b}}$, involved in the calculation of the modal sensitivities (Eqs. (29-32) and (35-36)) are calculated using complex step differentiation. The derivatives $\partial \mathbf{K} / \partial \rho_{\mathrm{b}}$ and $\partial \mathbf{M} / \partial E_{\mathrm{b}}$ equal zero. When assuming a known input $u$ (case 5), application of the ORC reveals that the two physical parameters $\left(E_{\mathrm{b}}\right.$ and $\left.\rho_{\mathrm{b}}\right)$, the modal damping ratios $\left(\xi_{m}, m=1, \ldots, 4\right)$, and the modal displacements and velocities are all MROM-observable. The same level of observability is obtained when the input is not present $(u=0$, case 6$)$. As such, it can be concluded that the identification of the stiffness and mass of the bridge deck does not require a measured and hence known input $u$.

\section{Conclusions}

This paper investigates the observability of modally reduced order models with unknown parameters. Since these models only approximate the dynamic behavior of the underlying dynamic system in a limited frequency range, their observability requires specific attention. A generic methodology is presented to account for the frequency-dependent model accuracy in an observability test. The result is applicable to any geometric or algebraic observability test for the case of a linear underlying system. In this work, the commonly adopted ORC algorithm is extended to 
address the theoretical observability of modally reduced order models. The model order reduction is performed by means of a recently developed quasi-static correction technique that can be applied in combination with state-space modeling. The methodology is illustrated for a 4 degree of freedom model and a detailed finite element model of an actual footbridge.

\section{Acknowledgments}

Kristof Maes is a postdoctoral fellow of the Research Foundation Flanders (FWO), Belgium. The financial support by FWO is gratefully acknowledged. The authors also thank Stijn François for the development of the finite element model of the Wetteren footbridge.

\section{References}

[1] R. Kalman, On the general theory of control systems, in: Proceedings of the First International Congress of IFAC, Moscow.

[2] M. Chatzis, E. Chatzi, A. Smyth, On the observability and identifiability of nonlinear structural and mechanical systems, Structural Control and Health Monitoring 22 (2015) 574-593.

[3] R. Hermann, A. Krener, Nonlinear controllability and observability, IEEE Transactions on Automatic Control 22 (1977) 728-740

[4] H. Nijmeijer, A. van der Schaft, Nonlinear Dynamical Control Systems, Springer-Verlag New York, Inc., New York, NY, USA, 1990.

[5] A. Isidori, Nonlinear Control Systems, Springer-Verlag New York, Inc., Secaucus, NJ, USA, 3rd edition, 1995.

[6] S. Diop, M. Fliess, Nonlinear observability, identifiability, and persistent trajectories, in: Proceedings of the 30th IEEE Conference on Decision and Control, pp. 714-719 vol.1.

[7] L. Ljung, T. Glad, On global identifiability for arbitrary model parametrizations, Automatica 30 (1994) $265-276$.

[8] A. Sedoglavic, A probabilistic algorithm to test local algebraic observability in polynomial time, Journal of Symbolic Computation 33 (2002) 735-755.

[9] S. Diop, Y. Wang, Equivalence between algebraic observability and local generic observability, in: Proceedings of 32nd IEEE Conference on Decision and Control, pp. 2864-2865 vol.3.

[10] M. Sain, J. Massey, Invertibility of linear time-invariant dynamical systems, IEEE Transactions on Automatic Control 14 (1969) 141-149.

[11] A. Martinelli, Extension of the observability rank condition to nonlinear systems driven by unknown inputs, in: Proceedings of the 23rd Mediterranean Conference on Control and Automation, MED 2015, Torremolinos, Spain.

[12] K. Maes, M. Chatzis, G. Lombaert, Observability of nonlinear systems with unmeasured inputs, Mechanical Systems and Signal Processing 130 (2019) 378-394.

[13] B. De Jager, The use of symbolic computation in nonlinear control: Is it viable?, IEEE Transactions on Automatic Control 40 (1995) $84-89$.

[14] X. Shi, M. Chatzis, M. Williams, Robust computation of the observability of large linear systems with unknown parameters, in: R. Caspeele, L. Taerwe, D. Frangopol (Eds.), Proceedings of the 6th International Symposium on Life-Cycle Civil Engineering, IALCCE 2018, Gent, Belgium, pp. 195-201.

[15] K. Maes, G. Lombaert, The influence of out-of-band modes in system inversion, Mechanical Systems and Signal Processing 115 (2019) 173-187.

[16] K. Maes, F. Karlsson, G. Lombaert, Tracking of inputs, states and parameters of linear structural dynamic systems, Mechanical Systems and Signal Processing 130 (2019) 755-775.

[17] R. Nelson, Simplified calculation of eigenvector derivatives, AIAA Journal 14 (1976) 1201-1205.

[18] C. Papadimitriou, E. Ntotsios, Structural model updating using vibration measurements, in: M. Papadrakakis, N. Lagaros, M. Fragiadakis (Eds.), Proceedings of the ECCOMAS Thematic Conference on Computational Methods in Structural Dynamics and Earthquake Engineering COMPDYN2009, Rhodes, Greece.

[19] Multiprecision Computing Toolbox. Advanpix, Tokyo, version 4.3.0.12074. http://www. advanpix. com.

[20] L. Trefethen, D. Bau III, Numerical linear algebra, Society for Industrial and Applied Mathematics, Philadelphia, PA, 1997.

[21] E. Reynders, D. Degrauwe, G. De Roeck, F. Magalhães, E. Caetano, Combined experimental-operational modal testing of footbridges, ASCE Journal of Engineering Mechanics 136 (2010) 687-696. 
[22] E. Lourens, C. Papadimitriou, S. Gillijns, E. Reynders, G. De Roeck, G. Lombaert, Joint input-response estimation for structural systems based on reduced-order models and vibration data from a limited number of sensors, Mechanical Systems and Signal Processing 29 (2012) 310-327.

[23] D. Dooms, G. De Roeck, G. Degrande, G. Lombaert, M. Schevenels, S. François, StaBIL: A finite element toolbox for MATLAB, Technical Report BWM-2009-20, Department of Civil Engineering, KU Leuven, 2009.

[24] H. Bachmann, Vibration upgrading of gymnasia, dance halls and footbridges, Structural Engineering International 2 (1992) 118-121.

[25] D. Anastasopoulos, M. De Smedt, L. Vandewalle, G. De Roeck, E. Reynders, Damage identification using modal strains identified from operational fiber-optic Bragg grating data, Structural Health Monitoring 17 (2018) 1441-1459.

[26] E. Reynders, System identification methods for (operational) modal analysis: review and comparison, Archives of Computational Methods in Engineering 19 (2012) 51-124. 